\title{
Aseptic Loosening of Total Hip Arthroplasty as a Result of Local Failure of Tissue Homeostasis
}

\author{
Jiri Gallo et al.* \\ Department of Orthopaedics, Palacky University, Teaching Hospital Olomouc, \\ Czech Republic
}

\section{Introduction (Gallo)}

Total hip arthoplasty (THA) is the most effective and safest method for treating severe degenerative, post-traumatic and other diseases of the hip joint. It is estimated that more than 1,000,000 THAs are performed each year globally. The incidence of primary THA increased in the period 1990 to 2002 in the USA from 47/100,000 to 69/100,000 (Kurtz et al. 2007). A similar study in Denmark reported an increased rate of THAs from 101/100,000 to 131/100,000 during the period 1996 to 2002 (Pedersen et al. 2005). More importantly, modelled future projections expect further increase in the need for THAs.

It is believed that THA can reliably relieve pain and improve function in the majority of patients for a period of 15 to 20 years or more postoperatively. On this basis, with the extension of THAs to a younger and generally more active population, the expected time of service of THAs would be insufficient and the number of revision surgeries would therefore increase during this time. The Kaplan-Meier ten-year revision-free survival estimates for younger patients range from $72 \%(95 \% \mathrm{CI}$ : $67-76)$ in Finland to $86 \%(95 \% \mathrm{CI}: 84,5-88,2)$ in Sweden (Corbett et al. 2010). Hence, $14 \%$ to $28 \%$ of such patients on average did not achieve a 10-year THA functioning without revision.

The main reason for late failure of THA is aseptic loosening accompanied by osteolysis followed by infection and instability of the THA that compromise more the early postoperative

\footnotetext{
*Yrjö T. Konttinen"1, Stuart B. Goodman², Jacob P. Thyssen³, Emmanuel Gibon", Jukka Pajarinen, Yuya Takakubo ${ }^{5}$, Peter Schalock ${ }^{6}$, Zygmunt Mackiewicz ${ }^{7}$, Eemeli Jämsen ${ }^{1}$, Martin Petrek $^{8}$, Rihard Trebse ${ }^{9}$, Andrei Coer ${ }^{10}$ and Michiaki Takagi ${ }^{5}$

${ }^{1}$ Department of Medicine and Department of Anatomy, Helsinki University Central Hospital, ORTON

Orthopaedic Hospital of the Invalid Foundation, COXA Hospital for Joint Replacement, Finland

${ }^{2}$ Department of Orthopaedic Surgery, Stanford University, USA

${ }^{3}$ Department of Dermato-Allergology Copenhagen University Hospital Gentofte, Denmark

${ }^{4}$ Department of Orthopaedic Surgery, Bichat Teaching Hospital, Paris School of Medicine, France

${ }^{5}$ Department of Orthopaedics, Yamagata University, Japan

${ }^{6}$ Department of Dermatology, Harvard Medical School, Massachusetts General Hospital, Boston, USA

'Department of Histology and Embryology, Medical University in Bialystok, Poland

${ }^{8}$ Department of Immunology, Palacky University Olomouc, Czech Republic

${ }^{9}$ Valdoltra Orthopaedic Hospital, Ankaran, Slovenia

${ }^{10}$ College of Health Care, University of Primorska, Izola, Slovenia
} 
period than the later one (Ulrich, Seyler et al. 2008). For this reason the above mentioned increase in rate of primary THAs is closely associated with increase in demand for revision THAs. The incidence of revision THAs in the USA increased from 9.5/100,000 to 15.2/100,000 between 1990 and 2002 and the projections by 2030 are even more impressive (Kurtz et al. 2007). With this in mind, research on the pathophysiology of aseptic loosening and osteolysis is of paramount importance and well justified.

This chapter provides a background on the current theory of aseptic loosening and osteolysis together with a brief overview of preventive measures.

\section{Overview of the current theories on aseptic loosening and periprosthetic osteolysis (Gallo, Goodman)}

The term "aseptic loosening" is closely related to the finding of gross mechanical instability at the interface between the implant and bone bed (Fig. 1). It is generally associated with pain and loss of function although it may also occur asymptomatically. Regarding the prevention the key question is what is the mechanism that first erodes the firm bond between implant and the bone bed.

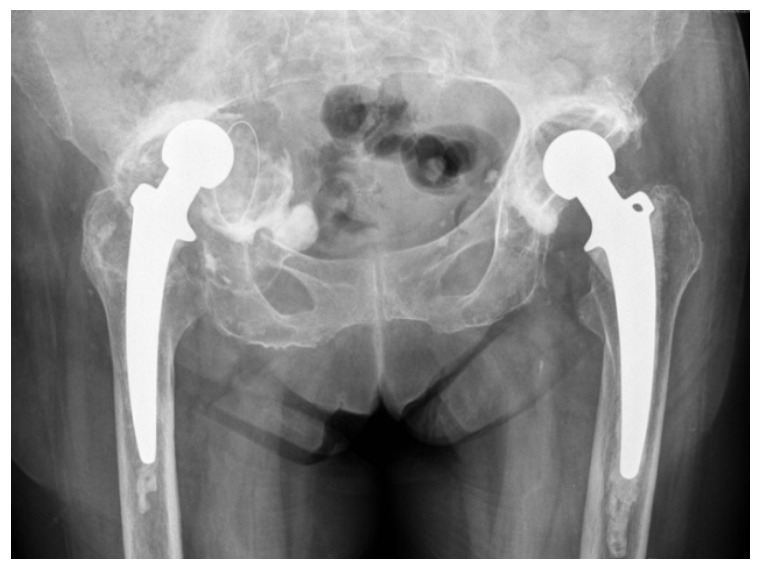

Fig. 1. Severe medial migration of the cemented cup at the right site in 86 year old female (17 yrs. postoperatively)

Aseptic loosening of total hip arthroplasty is hypothesised to be the result of a harmful combination of mechanical and biological events destroying the bond between the implant and bone bed. To date, a variety of host-, implant- and surgery-related factors have been delineated to explain the development of aseptic loosening and osteolysis. Some of these are accepted by the scientific community, others are the subject of intensive research, while yet others are only speculative having little or no clinical or research evidence.

There are specific factors that directly increase the risk of aseptic loosening while others induce conditions that increase the probability of developing aseptic loosening. A well acknowledged factor in preventing aseptic loosening is surgical experience and technique resulting in firm fixation and correct implant placement. Failure to achieve sound and longterm fixation of the implant to the bone bed is closely associated with premature aseptic loosening (Mjoberg 1994). Factors associated with the development of severe periprosthetic 
osteolysis significantly influence the rate of aseptic loosening as it is believed that severe bone loss can facilitate the process of destabilisation of the implant regardless of its original sound placement (Gallo et al. 2010).

\subsection{Mechanical theories on aseptic loosening}

Proponents of mechanical theories explain aseptic loosening and periprosthetic osteolysis to be the result of predominantly mechanical events with stress and strain being the major drivers of the disease. These refer to the stresses and strains affecting the implant-bone interface during each step until they overwhelm the firmness of the interface. On this basis, Mjöberg stressed the importance of the quality of the initial fixation because he and others had revealed the close association between early migration of implant and risk of premature failure of THA due to aseptic loosening (Mjoberg 1994). Unfortunately, there is no agreed threshold of migration above which loosening can be predicted. Further it is not possible to compare directly the migration data of different studies (Derbyshire et al. 2009). In addition, it seems that the majority of cemented cups migrate during the first postoperative year and thus the revision rate is poorly predictable one year postoperatively even in cases with significant migration (Aspenberg et al. 2008). For this reason, the "mechanical theory" should be revised to include at least the distinction between stable, osseointegrated prostheses and those in which osseointegration has not developed after the first postoperative year and exhibit radiolucency around the implant, and continuous migration resulting in loosening.

\subsection{Biological theories on aseptic loosening}

According to biological theory, aseptic loosening is the result of a series of predominantly biological processes. Of these, the most important is the complex adverse host response to prosthetic particles and by-products which produce particle disease. Willert and Semlitsch were the first to introduce the concept of aseptic loosening as a result of periprosthetic tissue reaction to massive amounts of prosthetic microparticles (Willert and Semlitsch 1977). The key concept in particle disease is that very small prosthetic particles (the size of micrometers and less) stimulate periprosthetic cells to express proinflammatory/ pro-osteoclastic cytokines and other substances that orchestrate increased accumulation/activity/survival of osteoclasts, and inhibit the osteogenic activity of osteoblasts. As a result, osteoresorption predominates over osteogenesis at each bone multicellular unit on the bone surface around the implant leading eventually to macroscopically observed bone defects. The degree of bone loss according to this concept is a function of number and depth of deregulated resorption sites.

The expansion of particle disease across the joint is facilitated by joint fluid that is abundantly synthesized by synovial-like macrophages and fibroblasts. Joint fluid contributes to conversion of M0 on M1 macrophages (Part 4.4). In addition, it washes the prosthetic microparticles from the articulating surfaces, mediates signal molecules and enzymes and delivers them to the bone. For this reason, particle disease can recur at new sites, contributing in this way to overall weakening of the bone-implant interface.

A question remains as to what other biological factors underlie the destabilization of the implant associated with severity of bone defects. Of these, aging of the bone bed (senescence), stress-shielding, and cumulative fatigue changes at the bone-implant interface may contribute to weakening of the implant-bone fixation. Eventually, these separately or 
together lead to degradation of the fixation interface and increase the probability of aseptic loosening. The ultimate pathway of aseptic loosening is undoubtedly of mechanical origin. For this reason, a comprehensive theory of aseptic loosening has been proposed involving both biological and mechanical factors.

\subsection{Comprehensive theory on aseptic loosening}

A valid theory requires consideration of both mechanical and biological factors in the pathophysiology of aseptic loosening and osteolysis (Gallo et al. 2002). The micromotions arising at the bone-implant interface during normal gait cycles induce a bone resorption which occurs before any wear debris particles enter the system. Following a number of "empty spaces" at the implant-bone interface are quickly filled by fibrous membrane. Repeated "compression" (stress) of the membrane during each step can induce proliferation of fibroblasts that synthesize an abundant extracellular matrix to adapt to the mechanical stresses and strains around an implant. According to this concept, the process of aseptic loosening is about sequential hypertrophy of the interface membrane (biological reaction to mechanical strain; Fig. 2). At a later stage, the hypertrophic membrane facilitates the delivery of particles, cytokines, enzymes and other molecules from the joint space to the bone bed interconnecting mechanical and biological factors.

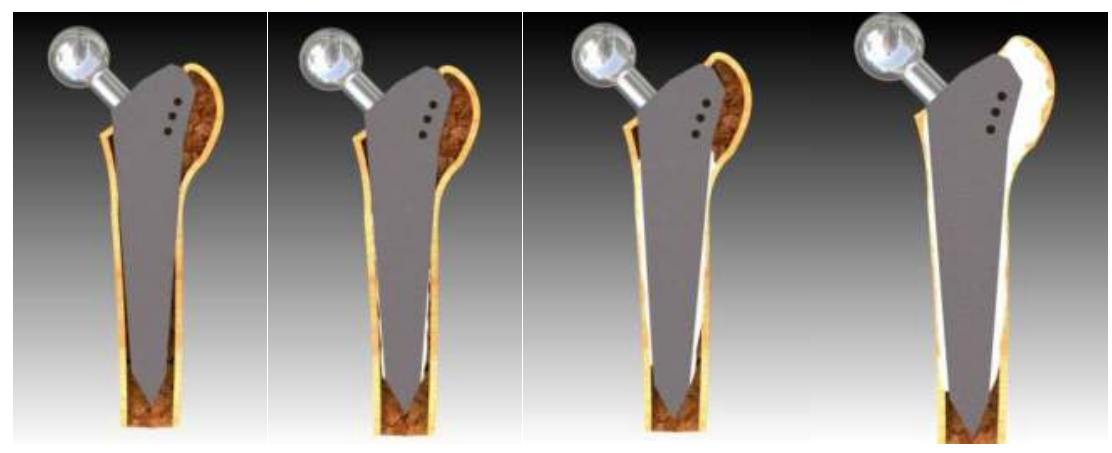

Fig. 2. Showing the process of gradual stem loosening as a result of hypertrophy of interface membrane (white) leading eventually to stem subsidence (reproduced by courtesy of Jiri Lostak, MD)

The mechanical environment can induce differentiation of mesenchymal stem cells via up-, down-regulation of mechano-sensitive genes and in this way it can influence tissue differentiation resulting for instance in excessive formation of fibrous tissue (Aspenberg et al. 2000). Interestingly, macrophages can be activated by cyclic pressure $(0.138 \mathrm{MPa})$ alone. In fact, there is significant increase in their expression of TNF- $\alpha$, IL- $1 \beta$ and IL- 6 when treated jointly with polyethylene particles and pressure (McEvoy et al. 2002). Other studies have demonstrate the relative sensitivity of macrophages to simultaneous application of PMMA particles and mechanical strain (Jones et al. 2006). With this in mind, one may conclude that mechanical conditions can directly regulate the proliferation and expression capacity of a particular cell group. Another scenario is based on evidence that high-pressure waves of the synovial joint fluid can induce bone necrosis (Fahlgren et al. 2010). An abundance of joint fluid is synthesized by synovial-like macrophages and fibroblasts inside the artificial joint as a response to inflammatory and secretory signals produced by particle disease. The degree 
of intraarticular pressure depends in part on the volume of joint fluid, changes in position of the joint, and volume/draining capacity of the joint space assuming that high-volume and effectively drained joint space are associated with lower intraarticular pressures and vice versa. Other factors that may be important, but are not readily analysed include at least interactions between implant and its surroundings in terms of local tissue homeostasis maintenance/distortion, favourable composition of joint fluid, type of lubrication, individual motion/stress pattern, and genetic predisposition to aseptic loosening.

\section{Local bone-soft tissue adaptations on an implant}

\subsection{What we know about the physiological reactions of the local tissues on the prosthesis and its waste products (Konttinen, Pajarinen, Mackiewicz, Takagi, Jämsen, Takakubo, Gallo)}

A lot of research has been done on aseptic implant loosening, but there are few studies about the physiological, adaptive host responses to joint implants with a long and painless life-in-service. Loosened implants are surrounded by a fibrous implant capsule (Fig. 2). Contrary, well (physiologically) fixed implants are not characterized by fibrous implant capsule, synovial-membrane-like interface membrane (SLIM) or aggressive granulomatosis. They are characterized by some other features described below.

\subsubsection{Physiological fixation of cemented implants}

Cemented implants are fixed to bone with bone cement, polymethylmethacrylate (PMMA). Hip implant surgery damages descending metaphyseal arteries during the resection of the femoral head and the intramedullary nutritional artery during the implant bed preparation, which contribute to ischemia, lowering of $\mathrm{pH}$ and bone infarctions (Willert et al. 1974; Konttinen et al. 2001).

Willert and co-workers made a cadaver study of well-fixed implants to describe the physiological three-phase response of host bone to cement fixed implants. Toxic and lipolytic PMMA monomers and the exothermic polymerisation cause a marginal rim of necrosis of bone trabeculae at the cement-bone interface. After three weeks and up to two years postoperatively signs of repair of the implant bed are seen. This is perhaps in part stimulated by a local release of bone growth factors from the bone matrix and activated osteoclasts. Necrotic bone and bone fragments are resorbed by osteoclasts and macrophages. Newly produced or extended, irregularly orientated bone trabeculae grow as a laceworklike structure into the relatively smooth necrotic-fibrin clot-fatty-fibrotic bone-cement interface, which contains cement plugs, some of which protrude to reach direct contact with peri-implant bone. After two years bone repair is followed by remodelling of bone trabeculae, which are separated from the cement surface by a 0.1-1.5 mm thick fibrous membrane. This membrane grows into the empty spaces between the rough or cracked cement surface and contains some polyacrylamide pearls (from non-polymerized PMMA powder) close to the cement surface surrounded (isolated) by foreign-body giant cells and granulomas. Trabeculae of the already remodelled peri-implant bone were in the longitudinal sections orientated in parallel to the cement surface (Willert et al. 1974).

This work was nicely extended by Jasty and co-workers, who studied serial horizontal sections of the proximal femoral bone that enclosed the cemented femoral component still in place in the implant bed. They confirm that the bone is remodelled and disclose a curious pattern for this remodelling. The cement mantles of the well-fixed implants are surrounded 
by an inner, secondary dense neocortex. There is bone ingrowth from the neocortex into the undulating surface of the cement mantle. This neocortex is connected to the outer, primary femoral cortex by new trabecular bone struts. These authors reported very little fibrous tissue intervening between the host bone and cement mantle around well-fixed implants (Jasty et al. 1990). They also suggest that loosening of the implant from the cement mantle (debonding) and fractures and fragments of the cement mantle coupled with ingrowth of a fibrous tissue layer between the implant surface and cement mantle may trigger loosening (Part 2.3). Based on this, stable, intact and firm cement mantle together with adaptive physiological bone remodelling response around are associated with a well fixed symptomless cemented implant.

\subsubsection{Physiological fixation of uncemented implants}

A second mode of fixation is based on the use of special design features and surface technologies enabling bone interdigitating ingrowth to the porous surface of the implant (osteoconductive ingrowth surfaces). Biomaterials and coatings can modulate bone ingrowth. Finally, successful fixation of uncemented implants depends also on the remodelling of already existing bone.

The initial fixation of the porous-coated implants to bone depends on the shape of the implant (e.g. wedge fit, threaded design), and/or the tight micromechanical locking (press fit, friction fit, scratch fit, interference fit) of an implant to the bone bed. The initial stability of the cementless implant is considered to be important for the secondary stabilization via bone ingrowth. The rough implant surface destined to fix the implant to bone has to come in contact with bone because bone cannot bridge very large gaps, perhaps exceeding 1-2 millimetres between the implant surface and bone. The micromotion at the bone-implant interface is first larger but diminishes below $\sim 40 \mu \mathrm{m}$ as bone grows to the surface of the implant. According to cadaver studies bone ingrowth can reach up to $50 \%$ in well-fixed implant while further penetration of the porous spaces available at the contact surface can reach $80 \%$. These values vary according to the quality of bone, type of implant/surface technology, and zone of implant (Engh et al. 1995).

Also this process is dependent on bone remodelling, i.e. formation of bone multicellular units (BMU) undergoing activation-reversal-formation (ARF) cycles, with net production of new cortical and trabecular bone. It is not quite certain, what is the micromotion at the boneingrowth surface area preventing bone ingrowth. In simulated stair climbing micromotion of cementless implants it is up to $280 \mu \mathrm{m}$ (Burke et al. 1991) which is close to 100-400 $\mu \mathrm{m}$ pore size commonly used in the porous coated implants still allowing bone ingrowth. The best implant-bone contact (bone ingrowth) is achieved in contact with compact, cortical bone characterized by Haversian channels. In other areas the porous surface may be in contact with ingrown cancellous, medullary bone without Haversian channels interdigitating rough implant surface. By this way the implant surface is connected with the endosteal side of the cortex (Engh et al. 1995).

\subsection{Does it exist something like long-term tolerance to an implant? (Takakubo, Pajarinen, Konttinen, Trebse, Coer, Mackiewicz, Takagi, Gallo)}

Assume that an experienced surgeon places well and firmly an appropriate implant. During a functioning of such THA soluble and insoluble particles of prosthetic biomaterials are deliberated from the prosthetic joint surfaces and the implant-bone interface has to withstand mechanical stresses on levels of multiple body weight. The question is on the mechanisms preventing premature destabilization of such implants. Although aseptic 
loosening and osteolysis are of multifactorial origin and many factors cannot be included into the failure analysis we believe that there is some place for a concept of host long-term tolerance to implant. Biological and non-biological circumstances (factors) can be associated with long-term painless and stable THAs.

\subsubsection{Genetic background of tolerance to an implant}

Tolerance may be defined as a state of progressively decreased responsiveness to a wear and corrosion particles as well as other stresses related to implant functioning. It is reasonable to hypothesize that the genetic background (host responsiveness) of the implant patient plays a role in loosening of or tolerance to the implant. Technically the approach taken has been to look for eventual single-nucleotide polymorphisms (SNPs) in candidate genes. SNP refers to single nucleotide base changes in the coding DNA at a particular site (natural sequence variants, alleles). Candidates for this approach have been sought among molecules, which according to the current understanding play a role in the pathomechanism of loosening. These candidates form three main classes, namely molecules regulating bone metabolism (influencing the fixation of an implant and maintenance of it), proteinases involved in collagenous tissue destruction (size of the effective joint space), and chemokines/ cytokines regulating inflammatory response to wear debris (aggressive granulomatosis). These processes are at least in part genetically regulated which opens theoretically the opportunity to screen the population preoperatively for risk patients. Current status in this field is described in part 4.7 .

In sensitized individuals even low local metal ion concentrations, such as cobalt and chrome, can cause T lymphocyte-mediated, delayed-type hypersensitivity (DTH) reactions. Metal ions can bind to self-peptides in the antigen presenting groove of the major histocompatibility complex (MHC) class II molecule altering self to altered-self, which is by the adaptive immune response recognized as non-self. Metal ions can bind to a protein altering its processing so that immunogenic cryptic (instead of tolerance-inducing dominant) epitopes are disclosed, causing DTH. They can modify MHC-II making it an immune-incompatible, "a foreign tissue-type antigen" (like in rejection of allogenic transplants) or they can activate the T-cell a bit like superantigens do. Tolerance to implant would require also immune tolerance against hapteninduced hypersensitivity reactions and in special cases it might be possible to test the implant patient-to-be pre-operatively for an eventual hypersensitivity with the help of epicutaneous patch or gamma-spot like tests (Part 4.5).

In a tolerant immunogenetic background wear particles are together with apoptotic bodies cleaned by M0 macrophages. Some co-factors may interfere with this tolerant host response to implant. Biomechanical loading (compression, tension, shear force) at the implant-bone interface may be one such cofactor. Occasional infections, microbial pathogen-associated molecular structures (PAMPs, e.g. lipopolysaccharide) and release of alarmins (e.g. high mobility group box 1) from damaged tissues (e.g. excessive biomechanical loading or ischemia) and necrotic or even activated cells can transform the M0 macrophage response into aggressive granulomatosis, which leads to peri-implant bone destruction. This in part, because both PAMPs and alarmins can opsonize wear particles. Various pattern recognizing receptors, such as Toll-like receptors (TLRs), mediate innate host responses and their SNPs may play a role here (Takagi et al. 2007; Pajarinen et al. 2010). In addition, primary and longterm fixation of the cemented and uncemented implants is dependent on the remodelling ability of the bone which is under genetic control. Peri-implant cells have to resorb damaged or necrotic peri-implant bone, repair it and then remodel it so that it maintains implant well 
fixed to the bone (Part 3.1). If fibrous interface and later synovial membrane-like interface or even foreign body-driven aggressive granulomatosus develop, activation-reversal-formation (ARF) cycles begin to dominate in bone multicellular units (BMUs) in peri-implant bone, contributing to the osteolysis and loosening of an implant.

\subsubsection{Other contributors to long-term tolerance of total hip arthroplasty}

Our knowledge on the factors associated with long-term functioning of a stable implant is in part based on comparison of tissues from patients with long-term survival of implant with tissues from those who suffer from loosening. In this line, it was for instance proposed that successful patients, in contrast to unsuccessful ones, could effectively clear the joint space from the wear and other particles and this way have diminished inflammatory stimulation (Zolotarevova et al. 2010). Also other patient-related factors, like gender, age, body mass index or even particular chronic medication (e.g. statins, bisphosphonates, nonsteroidal antiinflammatory drugs) could contribute to implant tolerance (Thillemann et al. 2010a; Thillemann et al. 2010b). Female gender seems to protect both the cemented and uncemented cups from early failure (Roder et al. 2010). Tolerance to implant seems to be improved with increasing age of the patients which could be explained not only by a decrease in activity level with no excessive cyclic loading in elderly patients but also a decreased responsiveness of the immuneinflammatory system might play some role (Ogino et al. 2008). Normal body mass index contributes to good implant tolerance at least in part via influencing bone remodelling around the implant and magnitude of mechanical stresses affecting bone-implant interface. A good general health and medication not adversely interfering with bone metabolism together with a good local quality of the bone could contribute to long-term maintenance of implantbone fixation. In this line, it is advisable to check the health status and medication of particularly elderly patients to optimize bone metabolism and bone strength. Targeting the dizziness and an individualized training program (muscle strength, coordination, and balance) together with interventions to the physical living environment of the patient, e.g. carpets, handrails, bedrails, illumination, doorsteps and various technology-related aids, could protect the implant from excessive loads.

Implant fixation and loading are interrelated and should be in balance for implant tolerance (Part 3.1). The number (cyclic regular loading) and type (on a smooth or declining surface, stairs, squatting etc.) of steps taken and any accidental or traumatic overshoots (not considered in computer controlled implant simulation studies) play a role. The number of steps taken can vary from 395 to 17718 steps per day, corresponding from 144175 to 6467070 steps per year (Schmalzried et al. 1998). In this way, it has been emphasized that the occurrence of polyethylene wear is a function of use, not time in situ (Schmalzried et al. 2000). Peri-implant bone microfractures and even pathological interface mobility can develop around already well-fixed implants as a result of too high loading. This secondary loss of stability stimulates bone remodeling (Takagi et al. 2001), and increases a risk for formation of fibrous interface around the implant. Fibrous tissue can further transform to synovial like-lining as a result of contact with synovial fluid (Part 2.2). Fibrous tissue formation is also promoted by the inert and passive nature of the metallic and polymeric materials used in joint replacements. Apart of the deteriorating effects of cyclic and erratic loading on development of fibrous interface, biomechanical loading affects also fluid flow and pressure in the lacuno-canalicular system. Detailed dynamic morphometric studies have shown high-turnover periprosthetic bone remodeling, immature bone formation and high density of osteocyte canaliculi in low-mineralized 
areas around loosening implants (Takagi et al. 2001). This can expose cells in the 3D osteocytes-network to too high or low shear stress (drag force) leading to their apoptosis and aseptic necrosis. Osteocytes subjected to high pressures in peri-implant bone die a programmed cell death, apoptosis. Too low intracanalicular flow or obstruction of fluid inflow through surface pores by wear debris induces osteocyte apoptosis/bone necrosis. Taken together, patients should be strongly advised against extremely strenuous use of the implant to obtain good implant tolerance, but this is naturally also a patient and implant specific situation.

\subsubsection{The effect of gliding pairs, implants and fixation}

It is believed that the outcome of THA depends on optimal combination of patient, surgery and implant related factors. In this line, evidence-based implant surgery creates basic conditions for inducing an implant tolerance. Material and implant selections can also influence the conditions under which the tolerance to implant may develop. Regarding particle disease, key features are amount of particles together with their specific functional activity. Generally, ultra-high molecular weight polyethylene (UHMWPE) particles are considered more stimulating than ceramic particles. The amount of polyethylene wear debris has been significantly decreased by the development of highly cross-linked UHMWPE (HXLPE) even though concern on its specific functional activity remains. Wear rate was decreased by $50-95 \%$ by the $1^{\text {st }}$ and $2^{\text {nd }}$ generation HXLPE compared to conventional UHMWPE. As a result, the risk for osteolysis was diminished with $87 \%$ for the $1^{\text {st }}$ generation of HXLPE liners compared to conventional UHMWPE liners (Kurtz et al. 2011).

In ceramic-on-ceramic (CoC) couples, with significant decrease of both the linear (5-300x) and the volumetric wear (30-2000x) in comparison to the metal on UHMWPE bearings $(\mathrm{MoP})$, the risk for severe osteolysis is even lower. In metal on metal (MoM) articulations the linear wear is 5-150x less and the volumetric wear 30-1000 x less than in the conventional MoP (Jin et al 2006) but concerns remain regarding induction of both the delayed-type hypersensitivity, e.g. Aseptic Lymphocyte-dominated Vasculitis-Associated Lesions (ALVAL). Taken together, if indeed a particle disease plays a significant role in loosening, these new materials might facilitate development of implant tolerance.

Information on the effects of specific implant design on tolerance to implant is obtained from finite element modeling (FEM), simulation, case-control cohort and arthroplasty register studies. FEM, experimentation in silica, has been used for implant design and testing, implant biomechanics and implant-induced responses, e.g. bone remodeling and stress shielding, but few studies have applied FEM to estimate the longevity of different implant designs, i.e. the long-term tolerance to implants (Korhonen et al. 2005). Another method suitable for implant testing is computer-controlled simulation of real implants (Lappalainen et al. 2003). Randomized controlled clinical trials are the best way to evaluate specific implants in clinical practice. Unfortunately, they are rare. More frequent small and medium size observational cohort studies performed in a single center or geographical area can be biased. In that case, the most reliable information on the performance of different implants can be obtained using arthroplasty registers. Such registry studies also support the practice involving combination of antibiotics in the bone cement and systemically to decrease the risk of revision, i.e. improve implant tolerance (Engesaeter et al. 2003). Implant register studies can also be used to help to identify the ideal patient to maximize implant tolerance. 


\section{Local host response to prosthetic wear debris}

\subsection{Hierarchy of immune reactions to prosthetic wear particles (Gallo)}

Aseptic loosening and osteolysis can be understood to be the result of chronic high-grade inflammatory disease induced by exposure of local tissue to massive quantities of prosthetic particles. For this reason predominantly investigated is the role of the immune system in its pathophysiology. Currently, there is a large body of evidence supporting the concept of the immune system acting major influence on particle disease. In this section, we will focus on the role of the different parts of the immune system in the process of aseptic loosening and osteolysis.

Basically the immune system consists of two tightly associated segments: the innate immune system and the adaptive immune system. The former consists of a set of pattern-recognition receptors (PRRs) that evolved to recognize pathogen-associated molecular patterns (PAMPs), triggering rapid and robust response to invading pathogens following their recognition. The adaptive immune system evolved to produce a highly specific response to unique antigen presentation after innate instructions are conveyed to the second line of host-immunity associated in particular with $\mathrm{T}$ and $\mathrm{B}$ cells. A distinction should be made between at least the inflammatory $T_{H} 1$ response inducing and perpetuating inflammation via expression of IFN- $\gamma$, IL-2, TNF-alpha and activation of macrophages, NK cells, cytotoxic lymphocytes, and anti-inflammatory $T_{H} 2$ and $T_{H} 3$ responses associated characteristically with expression of IL-4, IL-5 and TGF- $\beta$ and IL-10 cytokines, respectively (Matzinger 2007). From a functional point of view we can distinguish cells/pathways/molecules that trigger the immune response to wear debris and those that regulate host response, assuming the latter may play an important role in resolving inflammation and prevention of tissue damage.

The immune response is triggered after recognition of special surface motifs on invading pathogen (PAMPs) by PRRs. Intact pathogens can be composed of a number of PAMPs which induce activation of multiple PRRs simultaneously and, vice versa, different PRRs can recognize the same PAMP (Kumar et al. 2011). Currently, there is growing evidence that activation of PRRs is an important part of host adverse reaction against prosthetic particles (Lahdeoja et al. 2010). This could be explained by adherence of immunoreactive substances (lipopolysaccharides, lipoteichoic acid, fragments of bacteria, components of complement etc.) on wear debris particles. Some researchers have demonstrated that i) PAMPs adhere to the particles; ii) adherent PAMPs increase significantly the biological response to the particles; iii) PAMPs do this by activating their PRRs (Greenfield et al. 2010). In addition, particles deliberated from THA are coated immediately with serum proteins creating a protein-particle complex that may result in a damage/danger-associated molecular pattern picture irrespective of endotoxin (Sun et al. 2003). Engagement of TLRs leads to activation of the NF- $\mathrm{k} \beta$ signalling pathway with subsequent expression of pro-inflammatory cytokines, strongly boosting the immune response (Pearl et al. 2011). In the same way also other receptors (e.g. NOD-like receptor proteins) may participate in activation of the immune response to wear debris (St Pierre et al. 2010). Some of these are structurally involved in the cytosolic multi-protein complex known as the "inflammasome" which is considered a critical part of the inflammatory response. After appropriate stimulus, IL-1 $\beta$ and IL-18 convert from their inactive precursor molecules (pro- $1 \beta$ and pro-IL-18) into biologically active molecules that are then released from inflammasomes (Caicedo et al. 2009). From the clinical viewpoint, lipopolysaccharide has been detected in periprosthetic tissues without any other signs of infection in patients (Nalepka, Lee et al. 2006). In addition, some patients who underwent revision arthroplasty due to aseptic loosening had positive culture and/or PCR detected 
bacteria (Dempsey et al. 2007). In one recent study, it was even found that the extension of osteolysis correlated with proportion of positive sonication cultures (Sierra et al. 2011).

Participation of the adaptive immune system in host response to prosthetic wear debris is still a subject of controversy despite the fact that several reports have described lymphocytes in periprosthetic tissues, retrieved from patients with aseptically failed metal-on-metal (MoM) or non-MoM implants (Fujishiro et al. 2011; $\mathrm{Ng}$ et al. 2011). One recent study revealed increased serum concentration of cobalt and chromium in patients with MoM THA which positively correlated with increased proportion of HLA DR+ CD8+ T-cells in these patients (Hailer et al. 2011). In the same study, it was proposed that macrophages around THA could create haptenic metal ions in the context of self peptides (metal ion-altered self) as antigens in combination with MHC class II molecules, leading to T-cell priming. Weyand et al proposed antigen-recognition events when they characterised $\mathrm{T}$ cells in periprosthetic membranes (Weyand et al. 1998). They found identical T-cell receptor sequences suggesting identical antigen specificity and in addition, transcription of IL-2 and IFN- $\gamma$, indicating functional activity of at least some lymphocytes. Moreover, in this study, IFN- $\gamma$ transcription correlated with extension of bone loss. Sensitization to various heavy metals after THA is described elsewhere in this chapter (Part 4.5).

\subsection{Chemokines and cellular chemotaxis during the inflammatory response to wear particles from orthopaedic implants (Gibon, Goodman, Gallo)}

The biological reaction to polymers, ceramics and metallic wear particles is a non-specific foreign body and chronic inflammatory response. A type IV lymphocyte-driven hypersensitivity reaction involving a specific antigen has been found for a small percentage of cases with predominantly metal-on-metal bearing surfaces (Part 4.5). This section will focus on the inflammatory processes associated with wear particles from orthopaedic implants, emphasizing local and systemic cell signaling mediated by chemotactic cytokines called chemokines.

\subsubsection{The inflammatory reaction to wear particles}

Resident macrophages are amongst the first cells involved in particle-associated inflammation and initiate cell recruitment via the release of chemokines. Macrophage activation may occur with or without phagocytosis, by cell membrane contact with particles (Goldstein et al. 1975). Several receptors in the outer membrane of macrophages (e.g. CD11b, CD14, Toll-like Receptors and others) are involved in the activation of macrophages after contact with particulate debris. These receptors act through transmembrane proteins and different intracellular pathways and result in the release of cytokines, chemokines and other substances that induce cell recruitment. One of the most important intracellular pathways involved in signal transduction is the mitogen-activated protein kinase (MAP-Kinase) pathway. The MAP-kinase pathway then activates transcription factors such as the Nuclear Factor Kappa B (NFkB), which activates a cluster of genes for the production of pro-inflammatory cytokines, chemokines and related substances (Tuan et al. 2008). Besides macrophages, other resident cells are involved in the inflammatory process including fibroblasts, mesenchymal stem cells, osteoblasts, lymphocytes and others. Retrieval studies demonstrated that the periprosthetic cells produced high levels of pro-inflammatory cytokines and other factors including TNF $\alpha$, IL-1, IL-6, M-CSF, RANKL, and others (Part 4.3). 


\subsubsection{Accumulation of chemokines}

In addition to pro-inflammatory cytokines, chemo-attractive cytokines are also produced by different cell types involved in the inflammatory response at the bone-implant interface including: polymorphonuclear leukocytes, monocytes, macrophages, osteoblasts, fibroblasts and synovial cells. Monocyte chemoattractant protein-1 (MCP-1) is one of the most abundantly released chemokines and is an immediate early stress-responsive factor (Tuan et al. 2008). Macrophage inhibitory protein-1 (MIP-1 or CCL2) and interleukin 8 (IL-8) are also involved in these events.

MCP-1 is a C-C chemokine ( $\gamma$ chemokine subfamily) and its gene location in humans is 17q11.2. MCP-1 is primarily secreted by monocytes, macrophages and dendritic cells. With regards to bone, MCP-1 is also expressed by osteoblasts and osteoclasts. MCP-1 can bind to several receptors (CCR2, CCR4, CCR5) but binds preferentially to the CCR2. CCR2 has two isoforms: CCR2A and CCR2B; the expression of the different isoforms is restricted to specific cell types. Mononuclear cells and vascular smooth muscle cells express CCR2A whereas CCR2B is mainly expressed by monocytes and activated NK cells (Deshmane et al. 2009). MCP-1 regulates the migration and infiltration of monocytes, dendritic cells, memory T-lymphocytes and NK cells. Production of MCP-1 is under the direct control of the transcription factor NFKB.

MIP-1 belongs to the same subfamily of chemokines as MCP-1 (the C-C chemokine family), and is secreted primarily by activated macrophages and T-lymphocytes. Other cells may also secrete MIP-1. MIP-1 has two main isoforms: MIP-1 $\alpha$ (CCL3) and MIP-1 $\beta$ (CCL4). In mice, MIP- $1 \alpha$ and MIP-1 $\beta$ are encoded by one gene each, however in humans, MIP- $1 \alpha$ is encoded by at least three genes and MIP-1 $\beta$ by two, all located in chromosome 17 (Menten et al. 2002). Inducers of MIP-1 release are IL-1, IL-7, LPS and specific adhesion molecules (e.g. intercellular adhesion molecule-1, ICAM-1). High levels of MIP-1 $\alpha$ have been observed when macrophages from retrieved periprosthetic tissues have been challenged with titanium alloy and polymethylmethacrylate particles (Nakashima et al. 1999). The release of MIP-1 $\alpha$ increases the systemic recruitment of macrophages and has a paracrine effect enhancing IL-1 and IL-6 release (Cook 1996; Menten et al. 2002). Although MCP-1 has a specific receptor (CCR2), MIP-1 acts through several receptors (CCR1, CCR5), (Proudfoot 2002).

Interleukin-8 (IL-8) is a member of the CXC chemokine family, and also has the designation CXCL8. It is an early stress responsive chemokine and the main cellular sources are: macrophages, endothelial cells, epithelial cells, and cells of the mesenchymal lineage such as mesenchymal stem cells and osteoblasts. IL- 8 is a potent chemokine that induces the chemotaxis of neutrophils and monocytes/macrophages, and increases the mobility of osteoclasts (Baggiolini and Clark-Lewis 1992). Il-8 is also an angiogenic factor. IL-8 does not have a specific receptor but acts through the coupled $G$ protein receptors CXCR1 and CXCR2, which are receptors for other chemokines (e.g. GCP-2, NAP-2, Gro- $\alpha$ and others), (Proudfoot 2002). When osteoblasts are challenged with phagocytosable titanium particles, the IL-8 peak in gene expression occurs within 1-2 hours after particle exposure (Fritz et al. 2002). The biological mechanism of IL-8 expression involves first a protein tyrosine phosphorylation after contact with Ti particles and then activation of the pro-inflammatory

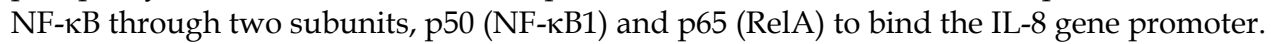

\subsubsection{Accumulation of cells}

Under the influence of chemotactic and other immunoregulatory signals, many different inflammatory and reparative cells migrate through local tissues regionally and systemically 
through the blood stream to the areas of particle generation. Recent murine studies involving continuous intra-osseous infusion of ultra high molecular weight polyethylene (UHMWPE) particles have demonstrated an increase in the systemic trafficking of reporter macrophages to the particle infusion site using non-invasive imaging (bioluminescence) and immunofluorescence microscopy (Ren et al. 2010). Furthermore, a decrease in bone mineral density has been observed within the UHMWPE particle-infused femora (Ren et al. 2011). This suggests that wear particles stimulate a systemic response, mediated by chemokines released locally. Recent unpublished studies in our laboratory have demonstrated that inhibition of the MCP-1-CCR2 chemokine-receptor axis can mitigate this response, including reversing in part, particle-induced bone loss.

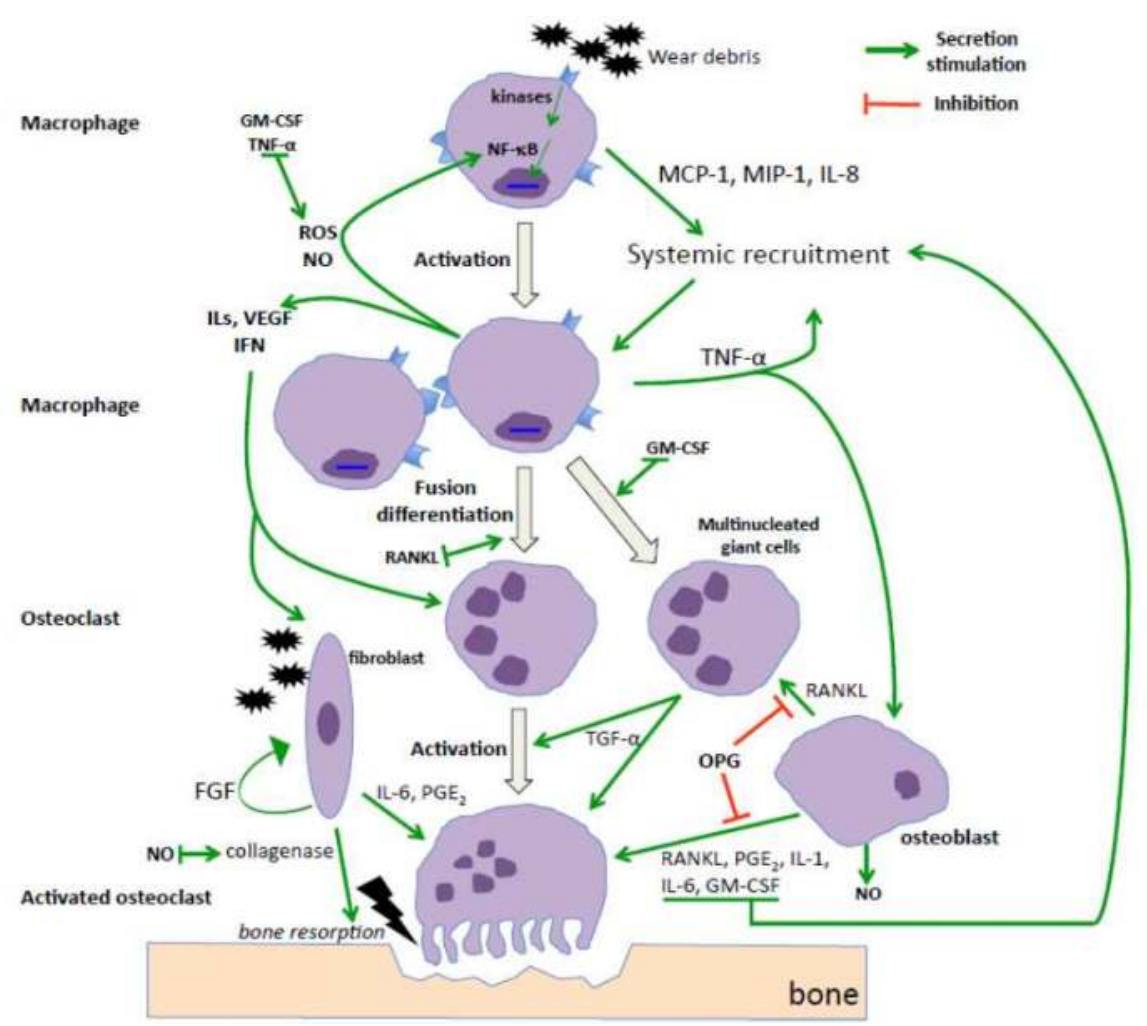

Fig. 3. Biologic reaction induced by orthopaedic wear particles on host cells; note the complex interaction of different cell types and factors to the presence of wear particles. Chemokines play a central role in cell recruitment. This diagram does not include the type 4 immune reaction to metallic wear particles.

$I L=$ interleukin, TNF $\alpha=$ tumor necrosis factor $\alpha$, GM-CSF = granulocyte macrophage colonystimulating factor, MCP-1 = monocyte chemoattractant protein-1, MIP-1 = macrophage inhibitory protein-1, ROS = reactive oxygen species, $N O=$ nitric oxide, $V E G F=$ vascular endothelial growth factor, NF- $\kappa B=$ nuclear factor $\kappa B$, IFN $=$ interferon, $R A N K L=$ receptor activator of nuclear factor $\kappa$ $B$ ligand, TLR = toll-like receptor, FGF = fibroblast growth factor, OPG = osteoprotegerin, TGF $\alpha=$ transforming growth factor $\alpha, P G E_{2}=$ prostaglandin $E 2$ 
After systemic and local cell trafficking to the inflammatory site, mononuclear macrophages may undergo a process called macrophage fusion. This results in polykaryons known as multinucleated "foreign body" giant cells which can be small (and contain 3-6 nuclei) or large (7-50 nuclei). Macrophage fusion is complex and requires the presence of specific cytokines and other factors (Part 4.4). The presence of foreign body giant cells has been widely demonstrated within the bone-implant interface (Goodman et al. 1989). Multinucleated giant cells have the capability to strongly express transforming growth factor- $\alpha$ (TGF- $\alpha$ ) as shown by Al-Saffar et al in their tissue retrieval study (Al-Saffar and Revell 2000). TGF- $\alpha$ is a key factor involved in angiogenesis, neovascularization and has been shown to impact both osteoclastic bone resorption and osteoclast-like cell growth and differentiation (Takahashi et al. 1986).

In vitro studies using murine bone marrow macrophages have shown that polymethylmethacrylate (PMMA) particles stimulate both RANKL expression, increase the number of osteoclasts and induce the formation of multinucleated giant cells (Clohisy et al. 2003). The authors also observed that PMMA particles activate the NF- $\kappa B$ and c-jun/AP-1 transcription factors, both of which mediate the osteoclastogenic effect of RANKL. Thus, after contact with orthopaedic wear debris, resident macrophages become activated and release proinflammatory cytokines, chemokines and other factors. MCP-1, MIP-1 and IL-8 release evokes the systemic recruitment of macrophages to the site of inflammation. Macrophage accumulation maintains the inflammatory process with release of pro-inflammatory cytokines that have paracrine and autocrine functions. Macrophage fusion induces the local release of TGF- $\alpha$ by multinucleated foreign body giant cells that facilitates the growth and differentiation of osteoclasts. Further cytokine release (TNF- $\alpha$, IL-1, RANKL and others) by resident macrophages, fibroblasts, and cells of the osteoprogenitor cell line maintains a heightened inflammatory state and promotes the growth and differentiation of osteoclasts. These interlinked pathways are summarized in figure 3.

\subsection{Cytokines and orchestrating of local adverse reaction to prosthetic particles (Pajarinen, Takakubo, Mackiewicz, Takagi, Konttinen)}

Cytokines are small $(15-25 \mathrm{kd})$ protein or glycoprotein messenger molecules that mediate intercellular (or even intracellular) communication and modulate immune reactions. They are produced, to some extent, by virtually all types of cells but by cells of the epithelial and hematopoietic origin as well as the cells of the immune system in particular. Typically cytokines are, after cell activation, secreted as soluble mediators into the extracellular space where they exert their function by binding to their specific high-affinity receptors located on the target cell membrane. Cytokine binding to its receptors induces receptor conformational changes and receptor dimerization or polymerization, binding of co-receptors in some instances and binding of adaptor proteins to the cytoplasmic tails, which leads to the activation of intracellular signalling cascades, activation or inactivation of specific transcriptional factors and finally up- or down regulation of gene expression. Some cytokines are not secreted but act in an intracrine (inside the cell) or juxtacrine (bound to cell membrane and requiring a direct cell-to-cell contact) mode of action while others are confined to the extracellular matrix (ECM) and released only when ECM proteins are degraded or damaged.

Cytokines control a very wide variety of different cell functions including cell growth, differentiation, migration, activation and survival. They are essentially involved into the development of blood cells (hematopoiesis) and not only in to the development and coordination of innate immune reaction and initial acute inflammation reaction but also to 
the activation and proper polarization of adaptive immunity, development of chronic inflammation as well as tissue regeneration and healing.

\subsubsection{Functional classification of cytokines}

Currently already more than 200 different cytokines are known. Cytokines can be classified into several broad categories by their functional properties. Such classification is at best a simplification as the biological functions of specific cytokines vary considerably depending on the context. For example, cell type and cells activation state as well as the presence of other activating or inhibiting signals derived from e.g. cell-to-cell and cell-to-ECM contacts and other cytokines can alter the final outcome of the cytokine signalling. This phenomenon is known as cytokine pleiotropy: one cytokine typically has multiple functions. Considerable redundancy exists in cytokine signaling, different cytokines can activate very similar intracellular signalling pathways and transcription factors and thus have very similar effects on the target cell. Cytokines can have synergy so that they enhance each other's signalling and the net sum of the effect is far greater than their additive effects. As cytokines typically regulate the secretion of other cytokines and the expression of each other's receptors, a complex and integrated network of cytokine functions is formed.

At the beginning of the inflammation reaction resident tissue macrophages are activated by exo- or endogenous danger signals recognized by different pattern recognition receptors (e.g. TLRs) to produce pro-inflammatory cytokines e.g. tumor necrosis factor alpha (TNFa), Il$1 \beta$ and IL-6. These cytokines activate and enhance the innate immune response, induce the secretion of anti-microbial molecules and other cytokines that refine the immune response, evoke systemic inflammatory response discussed above and importantly induce the expression of adhesion molecules in local microvascular beds in the process known as endothelial activation. Activated endothelium of post-capillary venules expresses selectin and integrin ligands, namely adhesion molecules (e.g. glycam-1, VCAM-1, ICAM-1), to which circulating and marginalized leukocytes effectively stick (capture and rolling), adhere and finally transmigrate from vascular compartment into the inflamed tissue. In unison with the pro-inflammatory cytokine production, macrophages, activated endothelial cells and other cells start to produce chemokines that further guide and activate transmigrated leukocytes to the site of inflammation along their increasing concentration gradient. The chemokines are discussed in more detail in part 4.2 of this chapter. Also interferons are secreted by the cells of the innate immunity at the early stages of innate immune reaction. They are traditionally classified in two groups having very distinct functions. Type I interferons ("anti-viral interferons"), including IFNa (13 subtypes), IFN $\beta$ and IFN $\omega$ (3 subtypes), are produced by macrophages after e.g. TLR recognition of viral structures (double stranded RNA or DNA). IFN $\gamma$, the only member of type II interferons ("immune interferon"), is secreted to some extend by macrophages and in greater magnitude by activated Th1 and NK-cells and is an important activating signal for macrophages which enhances their ability to secrete pro-inflammatory cytokines and kill phagocytosed bacteria. The initial strong pro-inflammatory response must be well regulated and typically proinflammatory cytokines are rapidly degraded, their actions are inhibited by soluble or solubilised receptor proteins (e.g. IL-1RA) and further counterbalanced by production of anti-inflammatory cytokines e.g. IL-4, IL-10, transforming growth factor- $\beta$ (TGF- $\beta$ ), which efficiently inhibit the production of pro-inflammatory cytokines and function of innate immunity. After the initial inflammatory reaction is resolved the tissue regenerative processes are rapidly engaged. Growth factors represent a diverse group of cytokines that 
regulate these tissue regenerative processes by modulating the growth and differentiation, of various cell types and tissues. Colony stimulating factors (CSF) typically control the myeloid development of monocytes and granulocytes, while epidermal growth factor (EGF), vascular endothelial growth factor (VEGF) and fibroblast growth factor (FGF) are secreted by macrophages and fibroblasts during regeneration of various tissues and they control e.g. formation of granulation tissue.

If the initial activation of innate immunity does not rapidly lead to the resolution of inflammation, the adaptive immune system is gradually activated by in particular professional antigen capturing, processing and presenting dendritic cells and macrophages that present MHC II-bound antigenic epitopes to the T-cell receptors (TCRs) of naïve CD4+ T-helper (Th) cells. The cytokine milieu that accompanies the antigen presentation process crucially determines the nature of the developing immune response (Th type $1,2,17$, Treg). It is in the field of bone biology useful to distinguish a group of cytokines that control the formation, activation and survival of bone resorbing cells, osteoclasts. The complete list of cytokines that have been shown to support osteoclastogenesis or their bone resorptive activity is quite extensive and includes e.g. TNFa, IL-1, IL-6, IL-7, macrophage-CSF (M-CSF), IL-11, IL-17, MIP-1a/ $\beta$, IFN- $\gamma$-induced protein-10 (IP-10) and monokine induced by IFN- $\gamma$ (MIG), bone (or body) morphogenic proteins 2 and 7 (BMP2, 7) and VEGF among others. Cytokines that inhibit osteoclastogenesis include IFN $\gamma$, IFN $\beta$, IL-4, IL-10, IL-13 and IL-18. Yet some cytokines play a dual role: low concentrations of TGF- $\beta$ stimulate osteoclast formation and high concentrations inhibit it. However, none of the above-mentioned cytokines seems to be absolutely necessary for osteoclast development (Theill et al. 2002). The most important cytokine system controlling the osteoclast formation and function is the RANKL/ RANK/OPG system (Boyle et al. 2003). Mesenchymal cells, e.g. osteoblasts, mesenchymal stromal cells and fibroblasts as well as activated T cells tightly control osteoclastogenesis by producing receptor activator of nuclear factor kappa B ligand RANKL, which is produced in both cell membrane-bound and solubilized forms. Binding of RANKL to its receptor RANK expressed on osteoclast progenitors or macrophages in the presence of M-CSF, leads to cell fusion and formation of mature bone resorbing osteoclasts. In addition mesencymal cells produce osteoprotegerin (OPG) which is a soluble decoy receptor for RANKL and acts to limit and regulate its function. A high RANKL/OPG ratio is thus considered to drive osteoclastogenesis. RANKL/RANK/OPG system seems to represent the final common pathway in osteoclastogenesis, and many of the above-mentioned cytokines that drive or inhibit osteoclastogensis act by regulating RANKL or OPG levels. In the presence of low levels of M-CSF, RANKL seems to both sufficient and necessary for the complete differentiation of osteoclast precursors into mature osteoclasts.

\subsubsection{Cytokines in aseptic loosening}

As the monocyte/macrophage and foreign body giant cells (FBGCs) are by far the most dominant cell type in the periprosthetic tissue, the wear particle activated macrophage has long been considered to play a pivotal role in the development of aseptic loosening.

Several studies over the years have demonstrated that monocyte/macrophages challenged in vitro with wear particles are activated to produce a wide variety of pro-inflammatory cytokines, chemokines and growth factors, including TNFa, IL-1 $\beta$, IL-6, IL-8, MCP-1 and MIP-1a/ $\beta$, VEGF, M-CSF, GM-CSF and even more so if particles are contaminated with bacterial products (Holt et al. 2007; Purdue et al. 2007; Goodman and Ma 2010). Also the shape, size, number, and biomaterial composition of wear particles seem to have an effect 
on the quantity and quality of the cytokines produced. This wear particle induced macrophage activation is possibly mediated by various types of cell surface pattern recognition receptors e.g. Toll-like receptors (Takagi et al. 2007; Tamaki et al. 2009) and likely involves the activation and action of transcription factor NF-kB (Lahdeoja et al. 2010; Pearl et al. 2011).

The mere wear particle stimulation of pure monocyte/macrophage cultures is not sufficient to drive osteoclastogeneis or foreign body giant cell formation, most likely due to lack of RANKL signalling. Further in vitro studies using other relevant cell types of the periprosthetic tissue have demonstrated that, to some extent, wear particles as such and especially pro-inflammatory cytokines effectively up-regulate RANKL production from periprosthetic tissue fibroblasts and osteoblasts as well as suppress osteoblast formation and function (Vermes et al. 2001; Mandelin et al. 2005a).

Consistent with in vitro findings, numerous studies of the explanted periprosthetic tissues and pseudosynovial fluid have demonstrated increased production of vast array proinflammatory cytokines, chemokines and growth factors including e.g. TNFa, IL-1 $\beta$, IL-6, IL-8, M-CSF, GM-CSF, MCP-1, MIP-1a as well as increased RANKL/OPG ratio favouring osteoclast formation (Mandelin et al. 2003; Holt et al. 2007; Purdue et al. 2007; Goodman and Ma 2010). Pseudosynovial fluid from aseptically loosened implants effectively induces formation of osteoclasts in cell culture conditions and OPG diminished this effect (Kim et al. 2001; Mandelin et al. 2005b). It should be noted however, that there is some discrepancy in these findings and, for example, the strong pro-inflammatory cytokines TNFa and IL-1 $\beta$ are not detected so consistently as e.g. IL-6, IL-8 or MCP-1, MIP-1a/ $\beta$ or RANKL possibly reflecting individual variations or different stages of the disease progression (Purdue et al. 2007). Because systemic inflammation reaction (e.g. fever, fatigue, cachexia) is not typically observed in patients with aseptic osteolysis, the observed low levels of TNFa and IL-1 might actually represent poorly understood aspects of particle induced chronic inflammation.

Studies conducted in animal models have further elaborated the role of pro-inflammatory cytokines and RANKL in the aseptic osteolysis. Several of these models have shown that implanted wear particles of various nature cause inflammation and increase RANKL/OPG ratio and osteolysis (Purdue et al. 2007). This reaction can be prevented or reduced by inhibiting TNFa signalling by using TNFa neutralizing antibody or by deleting TNF receptor (Childs et al. 2001). Similarly blocking of RANK signalling by RANK antagonist or OPG, or using mice genetically lacking RANK prevented experimental osteolysis in murine model of particle induced osteolysis (Childs et al. 2002; Ulrich-Vinther et al. 2002). Contradicting in vivo findings do also exist e.g. Taki et al. could not observe decrease in osteolysis in IL-1r and IL-6 or TNF double knock-out mice but concluded that osteolysis was likely mediated by other pro-inflammatory cytokines (Taki et al. 2007).

Taken together, following speculations can be made based on the detected cytokine profile of periprosthetic tissue. First, the definite majority of the cytokines detected are proinflammatory cytokines or chemokines secreted primary by cells of the innate immunity, which supports the essential role of activated macrophages in the process of aseptic loosening. Second, RANKL seems to be the most important end-point cytokine driving the osteoclast formation and loosening. Third, if only the cytokine profile of peri-implant tissues is considered, the role of adaptive immunity seems unlikely as both Th1 and Th2 signature and effector cytokines IFN $\gamma$ and IL-4 are not generally detected from the peri-implant tissues. Furthermore both IFNY and IL-4 effectively suppress the formation of osteoclasts. In this regard the possible role of Th 17 cells in the aseptic osteolysis seems an interesting line 
of further research as their signature cytokine IL-17 has been shown to favour osteoclastogenesis. Fourth, the nature of FBGCs remains somewhat elusive as morphologically sound FBGCs can be made in cell culture conditions using long term IL-4 stimulation, but as stated the IL-4 is not present in the periprosthetic tissue in any considerable amounts.

Based on the in vitro cell stimulation experiments and explanted tissue analysis, a following sequence of events can be postulated: wear particles activate macrophages, even more so if particles are coated by microbial products or alarmins. TLRs and other pattern recognition receptors of the innate immunity might mediate this wear particle induced cell activation. Activated macrophages produce pro-inflammatory chemokines, cytokines and growth factors which cause, via local endothelial activation and chemotaxis, further recruitment of monocytes and osteoclast precursors to the periprosthetic tissue. Pro-inflammatory cytokines, and to some extend also wear particles directly, drive the production of RANKL from mesenchymal cells and at the same time suppress the production of OPG and bone formation. Together with the pro-inflammatory cytokines they create an environment which favours ostoclastogenesis, bone resorption and finally prosthesis loosening. As the RANK/RANKL/OPG system seems represent the final common pathway in the proinflammatory cytokine mediated osteolysis, inhibition of RANKL might seem as a rational means to prevent aseptic osteolysis.

\subsection{Key cell players - macrophages, fibroblasts, lymphocytes, osteoblasts, osteoclasts (Gallo, Goodman)}

Histological and immunohistochemical studies of retrieved tissues have shown that when loosening is associated with osteolysis, the cellular profile is dependent on the method of prosthesis fixation (cemented or uncemented). Indeed, in the cemented group, tissue was more plentiful and often composed of highly vascularized fibrous tissue with a preponderance of macrophages and, to a lesser degree, T lymphocytes. With uncemented implants, tissue was less abundant and less cellular and more fibrous in nature. In addition to large numbers of fibroblasts, macrophages were also abundant but with increased numbers of all T-lymphocyte subgroups (Goodman et al. 1998).

\section{Macrophages}

Macrophages comprise a heterogeneous group of cells that play an important role in both the activation of the immune response and tissue homeostasis. They are derived from monocytes which arise from myeloid progenitor cells in the bone marrow. After maturation in bone marrow, monocytes are released into the circulation, then enter the tissues and differentiate into tissue macrophages (M0) that can survive for several months. Here, they can be activated by microbial/non-microbial agents (proinflammatory M1 macrophage activation), by IL-4, IL-13 etc. produced locally by mast cells and/or TREG lymphocytes (M2 antiinflammatory tissueremodelling macrophages), or by other signals (M2-like immunoregulatory macrophages). Suppression of cytokine signalling 1 (SOCS1) is a key determinant of differential macrophage activation and function influencing the overall macrophage balance in tissues together with other factors (Ma et al. 2003; Whyte et al. 2011).

Macrophages are considered the most potent phagocytic cells in tissues around the THA and there are believed to be able to phagocytise even non-opsonized particles, e.g. polyethylene, metal or polymethylmethacrylate. After phagocytosis of the wear particles (the "fuel of the particle disease"), the macrophages have to become activated to produce pro-inflammatory 
cytokines and orchestrate their microenvironment towards increased osteoclast differentiation and osteolysis. The mechanism by which macrophages recognize particles and become activated as a result is not fully understood. In this context it is interesting to speculate if perhaps excessive biomechanical loading, mast cell activation and/or tissue necrosis can release alarmins which could initiate expression of polarizing cytokines. Another option is that microbes or microbe-derived pathogen-associated molecular patterns could provide the danger signal initiating polarization of M0 to other macrophage subtypes (Tamaki et al. 2009). In fact, early in the adaptation process, tissue macrophages exhibit traits specific for the tissue in which they reside and, in the resolution phase of inflammation, they induce original tissue repair or fibrosis. It has been postulated that during initial or middle phases of tissue response on wear particle load M1 macrophage activation pattern could play a key role while end-stage of aseptic loosening could be associated with alternatively activated macrophages expressing predominantly chemokines such as CHIT1 and CCL18 (Koulouvaris et al. 2008).

Macrophages have their own weak bone-resorptive capacity but more importantly they are the cell reservoir for the generation of multinucleated giant cells (MGCs). Of these the multinuclear bone resorbing osteoclast derived from M0 macrophages is the most important consumer of bone. Induction of monocyte/macrophage-derived multinucleated giant cells has been demonstrated in response to hematopoietic growth factors (e.g. IL-3, granulocyte macrophage colony stimulating factor, GM-CSF), and IL-4. The role of adhesion molecules in polykaryon cell formation has also been reported, and mainly involves intercellular adhesion molecule-1 (ICAM-1/CD54) and the receptor CR3 (CD11b/CD18) expressed by multinucleated giant cells (Anderson et al. 2008). Furthermore, immunohistochemical analysis of tissues retrieved from aseptic loosened total hip and knee replacements has shown high levels of ICAM-1 and its receptor CD11b expressed by giant cells and phagocytic cells containing metal and polyethylene particles.

\section{Fibroblasts/ Fibrocytes}

Fibrocytes are mesenchymal cells that arise from monocyte precursors derived from blood. They express features of both macrophages and fibroblasts dependent on local factors participating in acute response to injury/surgery and also play an important role in regulation/ resolution of chronic inflammation (Reilkoff et al. 2011). Their increased occurrence in periprosthetic tissues long after the healing phase could be associated with disturbance of local tissue homeostasis.

About $30 \%$ of periprosthetic membrane is composed of fibroblasts and these cells exhibit a very high proliferation rate pointing on their activation state (Koreny et al. 2006). Fibroblasts are tissue resident cells that have the capacity to synthesize and remodel extracellular matrix. In this way they create the local tissue architecture and also develop a milieu in which particular homeostatic regulation (e.g. inflammation, repair) can occur (Buckley 2011). Thus, they could significantly influence the fate of a prosthesis because they govern the switch to resolving postoperative inflammation and formation of fibrous capsule (repair) during the initial postsurgery period (Anderson 2009). In fact, fibroblasts and fibrocytes residing in surface layers of the periprosthetic capsule are the first cell types that come into contact with prosthetic particles. Later, a series of interactions between immune cells (esp. macrophages) and fibroblasts could determine the overall adaptative capacity of periprosthetic tissues for wear debris. For this reason, fibroblasts in concert with other cells greatly influence the grade of periprosthetic inflammation. 
Aberrant expression of adhesion molecules, chemokines, cytokines, and their receptors could increase the inflammatory status, and vice-versa.

\section{Lymphocytes}

Local perivascular infiltrates of lymphocytes and even diffuse lymphocytes have been identified in specimens of periprosthetic tissue from both MoM and non-MoM implants (Fujishiro et al. 2011). Lymphocytes are a kind of leukocyte circulating in the blood. Precursors of both $\mathrm{T}$ and $\mathrm{B}$ cells are cloned in bone marrow but $\mathrm{T}$ cells require in addition, maturation in the thymus. The surface of each $\mathrm{T}$ cell displays thousands of identical $\mathrm{T}$ cell receptors and according to the presence of one or the other of two surface glycoproteins, they are designated as CD4 and CD8 T lymphocytes. The recruitment of lymphocytes into peripheral tissues where there is inflammation and/or damage is mediated by chemokines and adhesion molecules. In tissues, each B and $\mathrm{T}$ cell develops a specific response for a particular antigen interplaying complexly with innate immunity. As a result, different classes of pathogens stimulate differentiation of $\mathrm{CD}^{+} \mathrm{T}$ cells into effector $\mathrm{T}$ cells (Th1, Th2, Th17 subsets). The aim is to induce the best response to a particular danger signal without vast tissue damage. Therefore, an important role is played by the activity of regulatory $\mathrm{T}$ (Treg) cells which suppress inappropriate adaptive immune response via several regulatory mechanisms (e.g. TGF- $\beta$, IL-10).

T lymphocytes are key players in the process of local and systemic bone loss associated with inflammation (Djaafar et al. 2010). Importantly, direct cell-to-cell contact between activated $\mathrm{T}$ cells and pre-osteoclasts is critical for their maturation in osteoclasts via RANKL and other pro-inflammatory cytokines pathways. Natural killer cells (lymphocytes of the innate immune system) have been shown to be a principal tissue-infiltrating lymphocyte subset in patients with particle-mediated inflammation (Huss et al. 2010). However, despite the above-mentioned consequences, the role of the specific immune system in particle disease remains controversial.

\section{Osteoblasts}

The osteoblast is a mesenchymal cell with a primary role in bone formation and remodelling. To do this, they come in contact with other cell groups of different origins (including osteoclasts, macrophages, lymphocytes, bone marrow cells) and synthesize a variety of cytokines and chemokines. Such interactions likely play an important role under both physiologic and pathological conditions.

Given the problem is increased bone resorption around the implant, the interplay between osteoblasts and other cell groups likely creates an environment favouring increased osteoclast maturation and/or suppressed osteoblast activity. Investigated have been the following possible explanations. First, some types of prosthetic particles could be toxic to osteoprogenitor cells leading to slowing of their maturation. Second, wear debris could suppress the function of mature osteoblasts inhibiting their osteogenic capacity. Third, osteoblasts could phagocytose prosthetic particles and express pro-inflammatory and proosteoclastogenic molecules on their surface, contributing in this way to the severity of bone resorption. The same can be achieved by chronic inflammatory microenvironment that upregulates osteoblast synthesis of pro-osteoclastogenic molecules (Granchi et al. 2005; Fujii et al. 2011). Osteoblasts can contribute to the predominance of bone resorption at implant-bone interface also by specific interaction between them and $\mathrm{T}$ cells. Osteoblasts can express immunoreactive structures on their surfaces (superantigens) which directly stimulate $\mathrm{T}$ cells in their surroundings (Stanley et al. 2006). Activated T cells could express RANKL and M- 
CSF, required for maturation of osteoclasts and also for formation of other giant cells having potentially important implications for periprosthetic osteolysis and aseptic loosening.

\section{Osteoclasts}

Osteoclasts are multinucleated bone-resorbing cells that together with osteoblasts, play a pivotal role in bone homeostasis and bone remodeling. Osteoclast precursors derive from bone marrow as M0 macrophages circulating in blood until penetrating tissues and binding to the bone surface utilizing cell-specific signalling pathways. The mechanism of recognition of a particular place on the bone surface is not known. On the other hand, the integrins mediating the bonding of pre-osteoclast to the bone site are well described (e.g. $\left.a_{v} \beta_{3}\right)$. At the bone site, pre-osteoclasts fuse into multinucleated cells under the influence of fusion factors (e.g. dendritic cell-specific transmembrane protein; DC-STAMP) and then maturate in a particular milieu with the help at least of macrophage colony-stimulating factor (M-CSF) and receptor activator of nuclear factor kappa B ligand (RANKL), (Fig. 3). TNF-a and IL-1 potentiate this process, whereas a soluble RANKL neutralizer osteoprotegerin (OPG) inhibits it (Anderson et al. 2008). Indeed, RANKL/OPG ratio is considered a key regulator of osteoclastogenesis although in vivo, the RANKL-RANK interaction may occur in a direct cell-cell contact, e.g. between mesenchymal stromal cells and osteoclast progenitors or osteoblasts and pre-osteoclasts. A few giant osteoclasts form the cutting cone at basic multicellular units (BMU) in osteons (compact bone) and hemi-osteons (trabecular bone) that undergo resorption over a few weeks. The resorption space (lacuna) in healthy bone is filled in a few months by the closing cone composed of osteoblasts. However, under pathological conditions such as particle-mediated inflammation, the distortion of the coupling mechanism prevents closure of cone by osteoblasts. Hence, increased osteoclast bone resorption along with other mechanisms continues to create larger bone defects.

\subsection{Contribution of allergic and other metal ion mediated responses to loosening of a total hip arthroplasty (Thyssen, Schalock)}

Allergy and sensitization are frequently used terms, but their definitions should be clarified. Sensitization may be defined as "the process in which exposure to an antigen results in the development of hypersensitivity" whereas a definition of allergy could be "an altered body reaction, usually hypersensitivity, as a response to exposure to a specific substance". It is generally accepted that all metal prostheses release metal debris following wear and corrosion. This has been shown by measuring significantly elevated level of metal ions in hip arthroplasty patients; e.g capsular and periprosthetic tissues, the liver, spleen and lymph nodes, serum and urine (Hallab and Jacobs 2009). Metal ions may potentially activate the immune system, a response that is mainly adaptive rather than innate (Hallab et al. 2008). Typically, large particulate wear debris particles (more than $150 \mathrm{~nm}$ to $10 \mu \mathrm{m}$ ) are phagocytosed by cells including macrophages, which present antigens to circulating Tlymphocytes resulting in delayed type hypersensitivity reactions (Hallab and Jacobs 2009). Allergic complications following insertion of metallic orthopaedic implants are plenty and summarized in table 1 . In this section, we will discuss the possible association between metal allergy and loosening of hip arthroplasties.

Immune reactions described adjacent to metal implants include T-cell mediated type IV reactions but likely are multifactorial. Peri-implant reactions seem to be Th1 dominant. Hence, increased levels of IFN- $\gamma$ and IL-6 in metal hypersensitive joint arthroplasty patients (Hallab et al. 2008) as well as low IFN- $\gamma$ and elevated levels of IL-17 in nickel allergic patients with 
symptomatic joint implants but not in Ni allergic patients with well-functioning joint implants (Summer et al. 2010) have been reported. Tissues near metal devices in those with metal hypersensitivity may have elevated immune cells/markers including: CD3p Tlymphocytes, CD4p cells, CD11cp macrophages/dendritic cells, and cells with abundant MHC class II (HLA-DR) expression (dendritic cells), (Cadosch et al. 2009). Foreign body giant cell formation is often noted when phagocytosis of foreign particles by macrophages, including metals. Ingesting macrophages then secrete the proinflammatory cytokines TNF$\alpha$, IL-6 and IL1- $\alpha$ and $-\beta$ (Cadosch et al. 2009). At high concentrations, Ti and V cause production of superoxide anions in neutrophils and nickel ions break down neutrophil cell membranes at high levels (Kumazawa et al. 2002).

A novel mechanism for aseptic loosening of metal implants was suggested recently (Cadosch et al. 2010a). Osteoclasts mature and grow on titanium metals, leading to degradation, uptake and eventual release of the metal ions by the osteoclasts. This might explain increases in metal ion in systemic circulation, increased recruitment of osteoclast precursors via synthesis of specific chemokines, and also it can contribute to osteoclast differentiation. CCL17/TARC, CCL22/MDC, RANK-L, M-CSF and pro-inflammatory cytokines (CCR4) are elevated in the peri-implant environment (Cadosch et al. 2010b). This leads to loosening of the device as supporting surrounding bone is resorbed. In addition to being present in joint loosening, CCR4 is involved in the inflammatory reaction in cutaneous allergic contact dermatitis reactions (Vocanson et al. 2009). The link between cutaneous dermatitis and CCR4 is not proven, but is an area for further inquiry. While this evidence specifically address Ti metal, these same mechanisms could be relevant for other metal ions as well. Below, a description of the epidemiology of metal allergy and recommendation for clinical practice will be provided.

\begin{tabular}{|l|l|}
\hline Reactions & \\
\hline & \\
\hline Cutaneous: & Extra-cutaneous: \\
\hline Localized allergic (contact) dermatitis & Implant loosening \\
\hline Systemic allergic (contact) dermatitis & Pain \\
\hline Urticaria & Swelling/tumor formation \\
\hline Bullous reactions & Chronic inflammation \\
\hline Vasculitis & \\
\hline Impaired wound healing & \\
\hline
\end{tabular}

Table 1. Selected clinical manifestations of delayed type hypersensitivity reactions

\subsubsection{Epidemiology}

Metal allergy, as defined by a positive patch reaction to a given metal, is frequent in the general population since up to $17 \%$ of adult women and $3 \%$ of adult men are nickel sensitized (Thyssen et al. 2007). Chromium and cobalt allergy are less frequent and occur in about $1-2 \%$. Other prevalent causes of metal allergy include gold and palladium resulting in positive patch test reactions in about $10 \%$ of dermatitis patients albeit these are rarely clinically relevant (Faurschou et al. 2011). The prevalence of other metals, e.g. titanium, platinum, molybdenum, manganese and other has not yet been evaluated in general populations and are general infrequent in dermatitis patients. 
It has often been debated whether metal allergy increases the risk of developing delayed type hypersensitivity reactions, e.g. loosening of THA. The literature was recently reviewed and it was concluded that metal allergy might in a minority increase the risk of complications caused by a delayed type hypersensitivity reaction (Thyssen et al. 2011a). Also, we do not know how to identify the subgroups of metal contact allergic patients with a potentially increased risk of complications following insertion of a metal implant. However, it seems to be certain that insertion of metal bearings increase the prevalence of metal allergy as a review showed it was $25 \%$ among patients with well-functioning hip arthroplasties and $60 \%$ among patients with a failed or a poorly functioning implants (Hallab et al. 2001); considerably higher frequencies than general population estimates (Thyssen et al. 2007). Despite the higher prevalence in the latter patient group, it remains unknown whether the higher prevalence is caused by implant loosening or whether metal hypersensitivity results in loosening.

\subsubsection{Metal exposure}

In addition to metallic devices and prostheses, metal exposure is also related to intake of food and water, dental work, tattooing, and prolonged skin contact with metal objects. These exposures may all result in metal sensitization although oral intake sometimes may lead to tolerance. Oral intake of food and water as well as implantation seems rarely to result in allergic complications or clinical disease. However, skin contact with metal objects e.g. jewellery often results in skin sensitization and dermatitis at the site of skin contact. Several field studies have recently demonstrated that nickel release in concentrations that result in sensitization and dermatitis remains common in Europe and the United States (Thyssen and Menne 2010; Thyssen et al. 2011b).

\subsubsection{Patch testing}

Patch testing is widely used to establish a diagnosis of delayed type hypersensitivity to metals. The reproducibility of the patch test is high but allergen dependent (Brasch et al. 1994). Patch tests are typically applied to the upper back and occluded for $48 \mathrm{hr}$. Readings should be performed at least on day 3 or 4 and if possible on more than one occasion (Wahlberg 2006). Patch test studies have suggested that $24-34.5 \%$ of positive patch test reactions potentially are missed when readings are not performed beyond day 2 (Uter et al. 1996). Ready-to-use test systems such as the Thin-layer Rapid Use Epicutaneous (TRUE) test ${ }^{\circledR}$ generally have a good concordance with conventional patch test systems using e.g. Finn Chambers ${ }^{\circledR}$, except for cobalt (Lazarov et al. 2007). Patch testing intends to identify contact sensitized subjects by distinguishing between negative, irritant and allergic reactions. Thus, a valid positive patch test reaction typically requires a trained and experienced person that adheres to a set of valid criteria. Currently, the recommendations from the International Contact Dermatitis Research Group (ICDRG) dictate that homogeneous redness and infiltration in the entire test area is scored as a $1+$ reaction, homogeneous redness, infiltration, and vesicles in the test area are scored as a 2+ reaction, and homogeneous redness, infiltration, and coalescing vesicles in the test area as a $3+$ reaction (Wilkinson et al. 1970). 1+, 2+, or 3+ readings should be interpreted as positive responses indicating contact sensitization whereas irritant responses, doubtful (+?) responses, or negative readings should be interpreted as negative responses (Fig. 4). Clinicians should be aware that false positive and negative reactions may be encountered 
(Wahlberg 2006). Albeit there is raising concern that the patch test mainly measures cutaneous metal allergy rather than allergic reactions caused and effectuated by systemic immune cells, the patch test has worked well to establish an association between metal ion concentrations in the blood and urine, metal allergy and loosening of 1st generation metalon-metal hip implants. It is clear that a system test, e.g. the lymphocyte transformation test that measures immune reactivity in bone near structures or the blood may have a higher sensitivity and it is therefore warranted. However, no such test system is currently available for allergist and surgeons in most centres and countries.

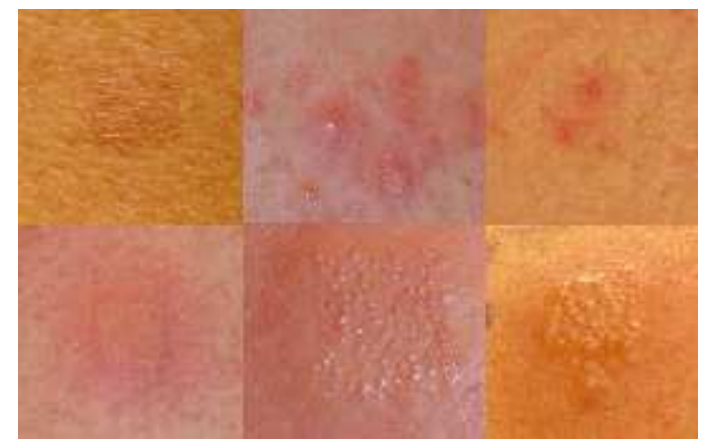

Fig. 4. Patch test reactions following 48 hours of occluded $5 \%$ nickel sulphate exposure. Upper line from left to right: irritative reaction, doubtful reaction (+?), and doubtful reaction $(+$ ?). Lower line from left to right: weak positive $(1+)$, moderate positive $(2+)$, and strong positive $(3+)$ allergic reactions.

\subsubsection{Other tests available}

In vitro tests such as the macrophage migration test, the lymphocyte blastic transformation test and the lymphocyte transformation test have mostly been used in research projects (Rasanen and Tuomi 1992; Bordignon et al. 2008). None of these have become routine analyses in the clinical setting, even when 2 or 3 methods have been combined. The lymphocyte transformation test measures proliferation of lymphocytes from peripheral blood in the presence and absence of a potential allergen after incubation for 7 days. The result is reported as a stimulation index (SI) and is therefore quantitative. The little use of such in vitro test is mainly explained by an overall limited availability, a small number of allergens that can be tested, and the need for fast examination of lymphocytes after sampling since they rapidly become incapable of proliferating. Nevertheless, the lymphocyte proliferation test has been claimed to better reflect immune reactions within the body whereas the patch test mainly rather reflect cutaneous reactivity. Thus, a linear correlation was identified between serum metal ion levels and lymphocyte reactivity suggesting that in vivo metal release is directly associated with metal contact allergy (Hallab et al. 2004).

\subsubsection{Clinical work-up}

It is difficult to make general principles for good clinical practice before and after surgery regarding delayed type hypersensitivity reactions to orthopaedic implants. Allergic reactions to metal ions released from implants are rare and at the same time metal allergy 
remains very frequent. This obviously makes it difficult to identify the few allergic subjects at risk before developing implant failure. Although it is a matter of controversy, it seems sensible to refrain from routine patch testing/allergy testing prior to surgery unless the patient has already had implant surgery with complications suspected to be allergic or a history of clinical metal intolerance of sufficient magnitude to be of concern to the patient or a health provider (Thyssen et al. 2011a). If a patient is strongly allergic to e.g. nickel or cobalt, one should consider inserting titanium based alloys. To demonstrate causal relationship between delayed type of hypersensitivity and aseptic loosening strict criteria (Tab. 2) should be followed (Thyssen et al. 2011a).

\begin{tabular}{|l|l|}
\hline Criteria & Description \\
\hline $\mathbf{1 .}$ & Histology consistent with a delayed type hypersensitivity reaction. \\
\hline $\mathbf{2}$. & $\begin{array}{l}\text { Positive patch test reaction to a metal used in the implant (often strong } \\
\text { reactions). }\end{array}$ \\
\hline $\mathbf{3 .}$ & Positive in vitro test to metals, e.g. the lymphocyte transformation test. \\
\hline $\mathbf{4 .}$ & Complete recovery following removal of the offending implant material. \\
\hline
\end{tabular}

Table 2. Objective criteria that support a causative association between metal release from the implant, metal allergy and delayed type hypersensitivity reactions such as implant loosening, malfunction, pain or chronic inflammation (from Thyssen et al. 2011a)

\subsection{Aseptic loosening as a result of local failure of tissue homeostasis (Gallo)}

The term "homeostasis" was coined by Claude Bernard in 1865 to describe the constancy of the internal environment as a condition of health. Metchnikoff has introduced the concept into immunology together with the term physiological inflammation to mean active maintenance of tissue harmony, tissue harmony (Medzhitov 2010). Given that the majority of patients profit from THA, it appears that low-grade periprosthetic inflammation occurring around "healthy" THA may be a normal adaptive response to the continual burden of prosthetic particles and mechanical stresses associated with implant use. For this reason, processes leading to osteolysis and aseptic loosening can be investigated as a problem of maladaptation.

In reality, no one knows what are the histological and immunological parameters associated with long-term "physiological" tissue homeostasis around THA, a baseline for true understanding of periprosthetic pathology. We can only translate data from models of THA (animal in vivo or in vitro) to the human situation (El-Warrak et al. 2004; Ma et al. 2009) and compare these with analyses of tissues from failed THAs. Unfortunately, tissues retrieved during revision surgery reflect late stages of the process when local homeostasis has long failed in the majority of cases.

What parameters are typical for physiological equilibrium between implant derived signals and healthy status of periprosthetic tissues in terms of maintenance of at least their architecture and function? The first and inevitable condition is stable interface between implant and bone which protects tissues from the deleterious effects of mechanical stimuli (stable equilibrium of implant-bone interface). The ideal condition is when the bone around the implant is protected from excessive mechanical stresses and strains and at the same time can undergo bone remodelling. The next critical step is the ability to resolve inflammation induced by surgical trauma together with prevention of biofilm formation on the prosthetic surface. Both the inability to achieve a steady very low-inflammatory status as early as possible and the 


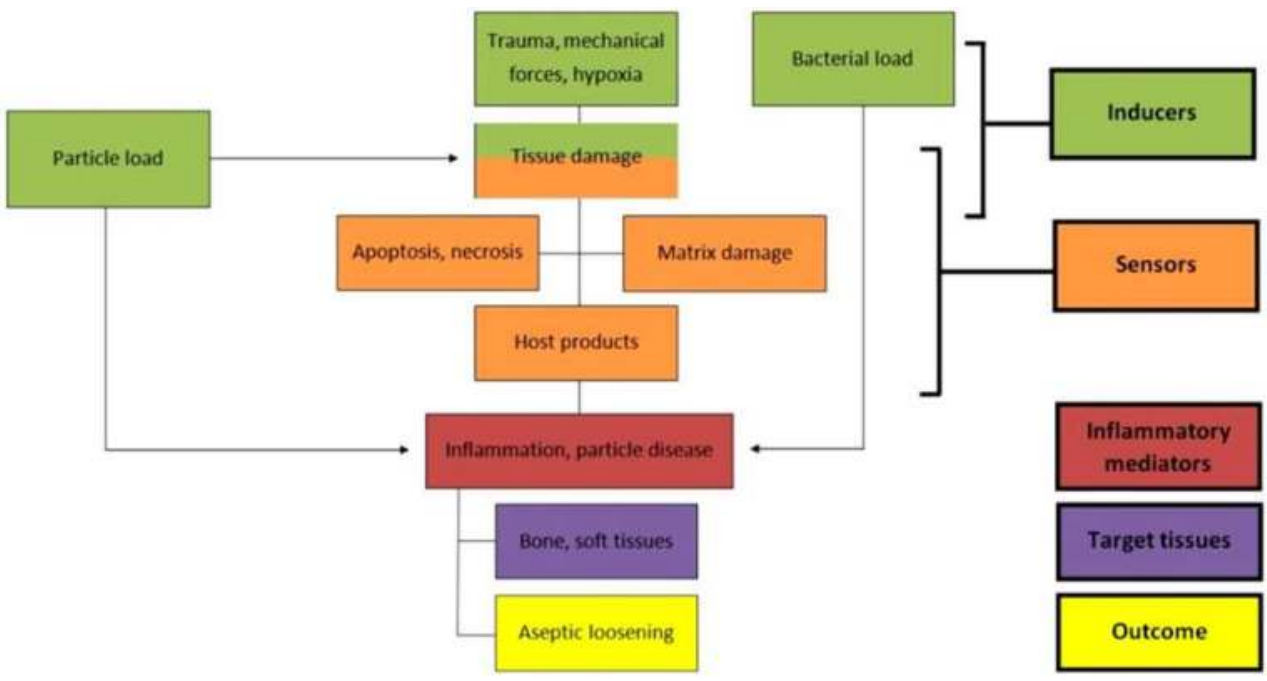

Fig. 5. Components of inflammatory response to a potential set of biological and physical insults associated with total hip arthroplasty (inducers of inflammation); host sensors detect the occurrence of inducers at tissues and their recognizing triggers expression of inflammatory mediators that affect the tissues where the inducers occur (according to Medzhitov 2010)

occurrence of microbial biofilm in the joint can maintain chronic inflammation which seriously compromises the local tissue homeostasis. The third critical step is the ability of tissues to counteract the continual delivery of soluble (ionic) and insoluble (particles) debris liberated from the prosthetic surfaces without increased inflammation and tissue damage. Prosthetic debris can induce a multitude of interactions which greatly increase the probability of chronic inflammation and predominance of osteoclasts over the osteoblasts at the bone-implant interface (Part 2.2). To avoid developing particle disease, there has to be much stronger regulation ability to overcome the above inflammatory and destructive processes. If these regulatory factors were known, new preventative and regulative measures could be proposed. The fourth critical step is the regenerative potential of the periprosthetic tissues that can restore tissue damage regardless whether this is of mechanical, biological or combined origin. Recently for instance, it has been proposed that a part of the inter-individual variance in the risk of early prosthetic migration (i.e. late aseptic loosening development) could be explained by patient-specific differences in the regeneration processes around the implant early post-operatively (Aspenberg et al. 2008). Taken together, when these steps concur, the probability of aseptic loosening is reduced and mutatis mutandis, if any of these steps is disturbed the route to aseptic loosening is open.

Normal tissue homeostasis is regulated by complex cross-talk between stem cells, their differentiated cells, the local microenvironment, and the whole organism (Perez-Losada and Balmain 2003). Basically, this depends on the right cell being in the right place at the right time (Buckley 2011). In periprosthetic reality this means, having sufficient number of functional macrophages, osteoblasts, fibrocytes-fibroblasts, and lymphocytes in a configuration that prevents development of chronic inflammation, fluid oversecretion, and 
increased accumulation of osteoclasts around the implant. The number of terminally differentiated cells is regulated by the mitotic activity of the stem cells in a poorly understood feedback interaction with local and systemic factors.

A very important role is also played by the local microenvironment which may favour development a particular cell line (Konttinen et al. 2005). In the case of THA, the inducers of inflammation can never be completely eliminated thus the tissues are under permanent activation load not only by mechanical stresses and particles but also by cytokines/ chemokines produced by cells previously activated by particles and/or microbial remnants. In fact, even under such conditions, the majority of periprosthetic tissues (patients) develop a steady state for a long time postoperatively. How is this possible? Can the tissues of successful patients develop something akin to "tolerance" (decreased responsiveness to the prosthesis-related stimuli)? Generally, to keep tissues in lowinflammatory steady state require as low load of inducers as possible and also very fine tuning of sensors together with strict control of the quantity and activities of effector molecules/cells (Fig. 5). On the other hand, the inability to resolve chronic high-grade inflammation can lead to irreversible tissue damage with restoration of different tissues (fibrous tissue instead of bone, formation of granulomas etc.).

\subsubsection{Control of prosthesis-related inducers}

Regarding the control of prosthesis-related inducers, most important appears to be reduction of the particle loads either using hard bearings or modern technologies of prosthetic surface treatment (Part 5.1). Of biological inflammation inducers, the most important roles are played by cell necrosis, extracellular matrix damage (both deliberating damage-associated molecular patterns, DAMPs), reactive oxygen species (ROS), hypoxia, and activation of PRRs.

Cell necrosis can affect both macrophages and other cell groups. It can be a result of strong foreign particle load (particles are per se undigestable, cytotoxic, genotoxic), hypoxia, ROS or unfavourable mechanical conditions. These stimuli can lead separately or combined to irreparable DNA damage and eventually cell death. Molecules released from dying cells together with breakdown products of the extracelullar matrix are considered important inducers of inflammation. In contrast, ingestion of apoptotic cells is associated with release of inflammation-resolving cytokines IL-10 and TGF- $\beta$ (Nathan and Ding 2010). From this, it follows that the ratio of necrotic cells to apoptotic cells around the implant could play a significant role in relation to periprosthetic homeostasis (Landgraeber et al. 2009).

Hypoxia seems to be a common feature in aseptic loosening. Bone-implant interface membranes can suffer from hypoxia because it is hypovascular, due to chronic hypoxiareperfusion injury of the interface membrane which is caused by implant loading. There is also increased oxygen consumption by local inflammatory cells (Santavirta et al. 1999). A hypoxic environment induces the expression of hypoxia-inducible factors (HIF-1a, HIF-2a) and some heat shock proteins that adapt the gene expression to oxygen availability within the hypoxic tissue. For this reason, decreased profileration is a fundamental physiological response to hypoxia in many cell types (Hubbi et al. 2011). Proliferation of progenitor cells is controlled by the hypoxia-sensitive Notch signalling pathway which maintains them in an undifferentiated state (Hilton et al. 2008). On the other hand, tissue macrophages are relatively well adapted to hypoxic conditions. This could at least partially explain their massive presence in periprosthetic membranes. Monocytes continue to differentiate into tissue macrophages even under severe hypoxia $\left(\begin{array}{lll}0.2 \% & \mathrm{O}_{2}\end{array}\right)$ and macrophages increase significantly their expression of HIF-1a mRNA and pro-angiogenic cytokine vascular 
endothelial growth factor (VEGF) as a response to chronic hypoxia. Hypoxia stimulates osteoclast differentiation, viability and activity, the latter predominantly via increased activity of hydrolytic enzymes including cathepsin $\mathrm{K}$ and in this way it could directly contribute to the extension of bone resorption around the THA. On the other hand, this effect depends on time and $\mathrm{O}_{2}$ concentration with reduction in number and activity of osteoclasts as a function of longevity and severity of hypoxia (Knowles and Athanasou 2009). Osteoblastic cell lines can exhibit decreased growth, differentiation and mineralization capacity under hypoxic conditions. Moreover, these cells predominantly induce angiogenesis via increased secretion of VEGF (Knowles and Athanasou 2009). An important role in the transduction of hypoxic signal to bone-implant interface may be played by osteocytes via increased expression of HIF and osteopontin leading eventually to osteocyte-induced osteoclastic bone resorption (Gross et al. 2005).

Activation of PRRs by occurrence of PAMPs in periprosthetic tissues can distort local tissue homeostasis at any time postoperatively (Greenfield et al. 2010). PRRs have a strong potential to trigger inflammation via several pathways including differentiation of more aggressive M1 and M17 macrophages, multinuclear foreign body giant cells, osteoclasts and granulomas (Anderson et al. 2008). In addition, it has been shown that polymeric alkane structures released during UHMWPE breakdown can directly activate PRRs (TLR-1, 2 signalling pathway) while UHMWPE particles phagocytosed by periprosthetic cells induce endosomal destabilization and inflammasome activation (Maitra et al. 2009).

\subsubsection{Tuning of inflammatory sensors and their response to prosthetic-related stimuli}

Currently there is no evidence supporting the concept of "adaptation of inflammatory sensors" to chronic stimuli associated with prosthesis. The following is therefore simply translated from knowledge of the response of macrophages to microbial stimuli (Medzhitov and Horng 2009).

After several hours or days of prosthetic-related stimulation, the expression of several hundreds of genes could be induced by macrophages, fibroblasts and other cell groups. These gene expressions determine cell response to the stimuli. Simultaneously, a set of regulators is co-activated to control intensity and extension of the local inflammatory response (negative regulators of inflammation). These can be distinguished as signal-specific regulators and gene-specific regulators. The first category consists of regulators that inhibit signal transduction by PRRs and other inflammatory pathways (e.g. IL-1R-associated kinase M, IRAKM; suppressor of cytokine signalling, SOCS - proteins). The second category comprises transcriptional repressors (basal repressors and inducible repressors) or other negative regulators that modulate gene expression (e.g. microRNAs, long-non coding RNAs). Although there is no evidence that currently supports the existence of "adaptors" on prosthetic stimuli, we believe they must exist. Assuming their role, it may be postulated that in the absence of a key part of the negative feedback loop the proinflammatory cytokines/chemokines develop severe inflammatory microenvironment associated especially with the predominance of activated osteoclasts over functional osteoblasts, and vice versa, effective negative regulation of sensors could protect from development of osteolysis and aseptic loosening (Parts 4.2, 4.3).

\subsubsection{Control of the effectors}

Generally, macrophages lie at the centre of the process called particle disease. Therefore there is a question whether activated macrophages can be deactivated and what are the 
mechanisms underlying the tolerance to chronic unsolvable signalling. Theoretically, there are at least two ways leading to deactivation of macrophages. The first is associated with decrease in stimulation signalling level (e.g. interferon gamma and other cytokines, lipopolysaccharides and other bacterial stimuli). The second could be associated with activity of tissue-resident cells inducing negative modulation of macrophage proinflammatory activities and macrophage apoptosis (Valledor et al. 2010).

Activated fibroblasts perpetuate inflammation via inappropriate expression of survival molecules leading to the retention of activated cells in affected tissues or ectopic secretion of chemokines supporting recruitment of new cells as a fuel for continuation of inflammation (Buckley 2011). They also express several bone-resorbing metalloproteinases and "osteoclastogenic" cytokines in periprosthetic membranes including M-CSF, VEGF or RANKL contributing together with suppression of osteoblast function, to functional predominance of bone resorption over osteogenesis (Koreny et al. 2006). Based on these observations, it seems inevitable that deactivated and quiescent fibroblasts can, like macrophages significantly contribute to the resolution of particle-induced inflammation. Recent studies have revealed that inflammation is not generic but contextual. Therefore, tissue-resident fibroblasts are able to switch the inflammation to the resolution. In this connection, it is important to know which mechanisms induce fibroblast anti-inflammatory and regeneration activities. Firstly, fibroblasts could act as a source of anti-inflammatory and regenerative cytokines such as IL-4, IL-10, FGF and several others. Secondly, fibroblasts can provide anti-inflammatory stromal microenvironment in the periprosthetic interface membrane involving a plethora of cell-to-cell and perhaps also paracrine interactions (Buckley 2011).

Lymphocytes together with other cells enhance osteoclast differentiation; stimulate formation of foreign body giant cells and exhibit many other activities contributing to tissue damage. On the other hand, they have several pathways by which they can "shift" the immune response in favour of $T_{H} 2$ and $T_{H} 3$ instead of $T_{H} 1$ and $T_{H} 17$ responses or delayed type of hypersensitivity (Matzinger and Kamala 2011).

Until now, we have assumed that the immune system alone dictates the direction and fate of periprosthetic tissues after stimulation by prosthetic derivates. However, this concept does not reflect the reality of the situation. Currently discussed is the concept of tissue-appropriate immunity, stressing the role of tissues in control of the effector mechanisms that prevent selfdestruction (Matzinger and Kamala 2011). On this basis, tissue-resident cells might influence the response to stimulus in order to maintain the health of affected tissues by modifying cell activities and via soluble anti-inflammatory factors. Unfortunately, no such strategy is available currently in clinical practice to modify the above processes and induce/maintain localized tissue homeostasis around THA.

\subsection{Individual susceptibility to aseptic loosening/ development of periprosthetic osteolysis (Gallo, Petrek)}

High variability in survival of THAs, aseptic loosening/size of periprosthetic osteolysis has been observed between individuals with similar polyethylene wear rates. For this reason, the question is how to explain such a degree of inter-patient variability? This could be caused for example, by differences in implants (e.g. type of bearing material, sterilisation method, shape of prosthesis, surface technology etc.), variations in surgical technique (orientation of implants, final quality of bone-implant interface, protection of bone bed from joint fluid etc.), and variations due to patient-related factors (e.g. age, co-morbidities, level of activity and differences in mechanical loading). In addition, genetic factors can contribute to 
the risk for aseptic loosening (Fig. 6). Engh et al estimated that both wear and patient propensity to osteolysis might together account for $53 \%$ of the variance in total area of osteolysis (Engh et al. 2011).

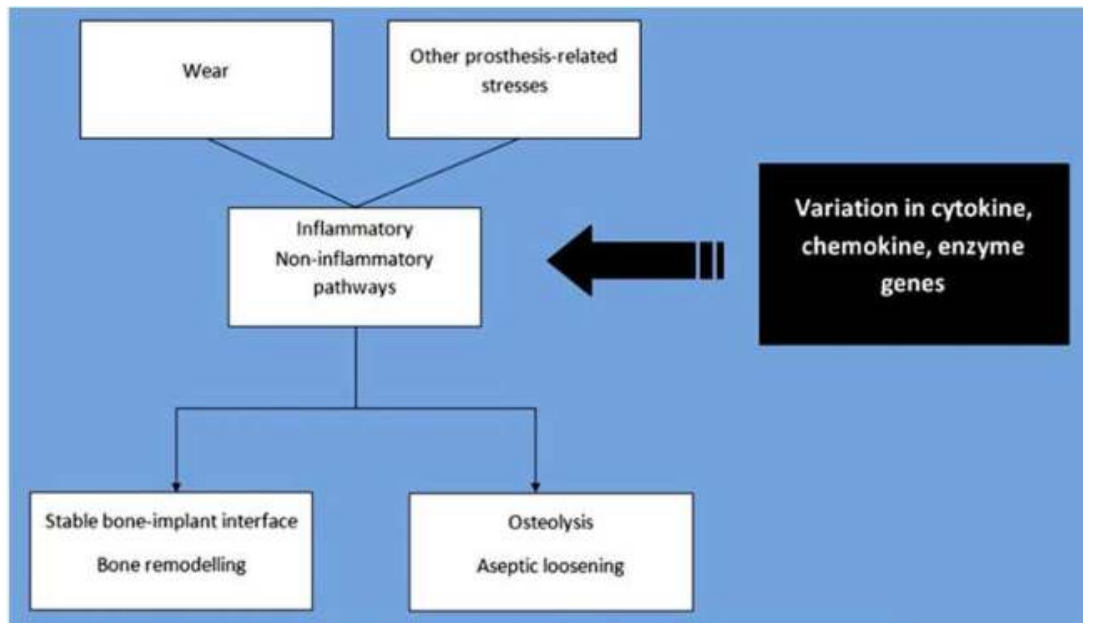

Fig. 6. Functional variation in genes for cytokines (other molecules) could influence the rate of aseptic loosening via contribution to severity of bone resorption around the implant

\subsubsection{Methods for investigating genetic susceptibility to aseptic loosening/ periprosthetic osteolysis}

The question is which genes (genotype) could influence the fate of implant in terms of premature aseptic loosening/ periprosthetic osteolysis (phenotype). A gene is traditionally defined as a segment of DNA encoding a protein. Single nucleotide polymorphism (SNP) is a common form of variation in the human genome implicating that a single base change in the DNA sequence could influence the amount/functionality of secreted proteins. With the sequencing of the human genome, it has been recognized that SNPs occur about once every 1000 base pairs (Dupuis and O'Donnell 2007). The key problem is how to distinguish the gold (functional polymorphisms) from fool's gold (unimportant variants). By genotyping a large number of SNPs in a large number of patients there is a good chance of detecting those that are significantly associated with the target disease. Unfortunately, epigenetic factors and further DNA sequence variants such as copy number variants create other sources of variation between individuals. In addition, there are several non-coding small regulatory molecules (e.g. miRNAs, siRNAs) that significantly influence the process of translation of genes into effector proteins.

According to the number of genes participating in the pathophysiology of a disease and the size of their effect, polygenic predisposition assuming small/ marginal effects of many genes fits much better to the model of THA failure than the oligogenic model with two or more major gene (locus) effects. In this line, the odds ratios of 1.2 to 1.6 are required for differentiating true effects of individual genetic variants on a complex disease from the potential impact of bias (Ioannidis et al. 2006). Studies reporting weaker individual size effects are unlikely to be used for predicting a target condition; on the other hand, a combination of several even weaker genetic variants (especially at multiple loci) can increase the chance for reasonable prediction. 
Genetic variation in patient-specific response to THA can be determined by several methods. For example, genetic association studies investigate possible association between the occurrences of phenotypic traits and candidate genetic variants using special rules and computational algorithms. In the case of genome-wide association studies the effects of up to 500,000 SNPs are analysed simultaneously, giving rise to an initial set of promising susceptibility genes that are further tested in a separate replication study.

When dealing with a genetic - environmental interaction it is necessary to test simultaneously cases, controls exposed to the same key pathogenetic factors as the cases but do not exhibit the risk phenotype, and the population control. In the case of aseptic loosening/osteolysis it is however rather difficult to recruit completely comparable cases and controls making it hard to address the question of individual susceptibility to these complications. In addition, identifying and characterizing the targets (mechanisms/molecules) for a genetic-association study is a key prerequisite for genetic investigation, and vice versa, inaccuracy in understanding the true pathophysiology of aseptic loosening/osteolysis compromises their outcomes. Therefore, the initial step of any genetic-association study should be choice of potentially valuable candidate genes followed by their validation in a candidate-gene study (Chanock et al. 2007).

\subsubsection{Current status of the research}

Wilkinson et al were the first to publish a study on the association between polymorphisms in the gene encoding for TNF alpha and risk of periprosthetic osteolysis in THA (Wilkinson et al. 2003). After this introductory work several papers were published nominating other molecules as candidates involved in the processes of aseptic loosening/ osteolysis. Structurally and functionally, these include receptors, intracellular mediators, enzymes, cytokines and other proteins. A recently published systematic review on genetically determined susceptibility to aseptic loosening of THA, revealed several areas of potential agreement (SNPs of TNF-238A allele, IL1RA +2018C allele, polymorphisms in genes for IL6, MMP-1 etc.), but also several sources of heterogeneity between studies, showing the need for large multi-centre prospective studies that should provide stronger evidence for genetic predisposition to THA premature failure (Del Buono et al. 2011). As a result, further progress in the field of genetic risk of aseptic loosening/ osteolysis will require both sophisticated research strategy and statistics (e.g. improved risk-analysis models) to overcome a series of challenges. Fortunately, as experience accumulates, there is increased interest of orthopaedic specialty in the outcomes of such research.

\section{Prevention of aseptic loosening/ periprosthetic osteolysis (Gallo, Goodman, Konttinen)}

\subsection{Biomaterial solutions}

Based on the pathophysiology of periprosthetic osteolysis and aseptic loosening, there is clear that advances in biomaterials can improve at least partially the survival of THA by reducing the extension and intensity of particle disease. Here we focus only on bearing surfaces, although the material of implant and surface technology also influence the resistance against osteolytic membrane and joint fluid expansion.

\subsubsection{Advances in polyethylene}

The wear of ultra high molecular weight polyethylene (UHMWPE) is a major contributor to premature failure of THAs. For this reason, a number of years ago, it was questioned 
whether this material should be retained or withdrawn (Amis 1996). Fortunately, over the recent decades, several processing techniques have been developed to improve the mechanical and tribological performance of UHMWPE. More recent technologies involving high degree cross-linkage of the linear chains of UHMWPE (highly-cross linked polyethylene, HXLPE) have been incorporated into clinical practice for several years. These also differ from company to company according to method used for the elimination of residual free radicals that could lead to oxidative degradation of bearing surface (heating, infiltration of vitamin $\mathrm{E}$ etc.). In comparison to ceramic-on-ceramic (CoC) THA, the advantage of HXLPE is greater variety in terms of head sizes and offset. Also the cost of HXLPE is substantially lower than that for hard bearing surfaces. On the other hand, there remains a concern about the risk of fatigue fracture of acetabular liners even though the cases reported were made predominantly of first generation HXLPE (Furmanski et al. 2009). Little is known on the biological activity of HXLPE particles. It was demonstrated moderately increased specific biological activity in comparison to particles of conventional polyethylene (Illgen et al. 2009). Despite that, there is growing evidence that the use of modern HXLPE bearings decrease significantly wear rate and by this way may reduce the risk of premature osteolysis development although the majority of studies report outcomes with less than 10 years follow-up (Kurtz et al. 2011).

\subsubsection{Advances in ceramic bearings}

The amount of prosthetic particles can be significantly reduced using CoC bearings in THA. Ceramics, in general are very hard materials with excellent biocompatibility. In addition, use of third generation ceramic bearings, reduces the predicted risk for fracture of ceramic bearings to approaching nil. Currently there is enough evidence supporting the further use of CoC bearings in the case of THA (Hannouche et al. 2011). Concerns remain about the "squeaking" of ceramic implants, impingement of the femoral neck on the edge of ceramic liner and microseparations of ceramic surfaces.

Oxinium is a $\mathrm{Zr}-2.5 \mathrm{Nb}$ alloy that has been oxidized at elevated temperature to grow a zirconium oxide ceramic layer on the surface of the implant. Such surface treatment increases the wear resistance of the implant. However, there is only limited clinical experience with Oxinium ${ }^{\mathrm{TM}}$ femoral heads in THA.

\subsubsection{Advances in metal}

Bearing surfaces made of cobalt, chromium and molybdenum alloys are currently a subject of controversy because of their biological consideration. The main reason is that metallic wear particles can induce implant hypersensitivity, ALVAL (Part 3.2.2), may contribute to tissue necrosis and also to induction and perpetuation of periprosthetic particle disease (Watters et al. 2010; Basko-Plluska et al. 2011). In addition, the levels of metal ions in serum and urine also can be elevated for a long time in patients with stable and functional implants in comparison to controls without metallic implants. Although long-term data are not available, the International Agency for Research on Cancer (IARC) has classified implanted foreign bodies consisting of metallic cobalt, nickel and chromium in Group 2B defined as possibly carcinogenic in humans (McGregor et al. 2000). The main concern is that exposure of human cells to cobalt and chromium may induce chromosomal aberrations and damage DNA, potentially leading to carcinoma (Beyersmann and Hartwig 2008). From a purely epidemiological view, the current evidence for clinical carcinogenetic effects of metal-onmetal THAs is limited and rigorous long-term studies are needed to answer the above question. Taken together, above potential consequences from the clinical usage of bearing 
surfaces made of cobalt, chromium, molybdenum alloy should be discussed with the patient prior to his/her surgical procedure.

\subsection{Design solutions}

THA has to withstand repetitive mechanical loading and long term exposure in the human body without compromising the integrity of either implant or bone-implant interface (Tarala et al. 2011). Engineers can help to prevent, at least partially, the development of periprosthetic osteolysis and aseptic loosening by creating a design and surface characteristics that increase the resistance of implant-bone interface to osteolytic granulomas and preventing access of joint fluid to the bone bed. The current evidence is in favour of bone remodeling being responsible for long-term bone-implant fixation. In this line, more biologically active prosthetic surfaces have been introduced into clinical practice (e.g. porous metals, hydroxyapatite coating etc.). Currently, multifunctional surfaces are tested with the aim of improving osseointegration and preventing the formation of biofilm. Recently, attempts have been made to coat implants with mesenchymal stromal (stem) cells (MSCs). The hypothesis is that such cells could enhance bone formation and subsequent implant fixation via intramembranous or endochondral ossification. However, the eventual role and fate of endogenously mobilized or exogenously delivered MSCs in implant fixation is still poorly known.

\subsection{Patient selection}

The final outcome of THA depends on a large number of factors including the design of the implant, surgical technique, and patient selection. Currently there are no scientifically proven criteria for matching individuals to particular implant. At the time of surgery, the decision should involve the age of the patient, his/her local bone status and overall health condition enabling rough estimation of time in use for the THA. Based on current best evidence, younger more active patients have several options to minimize wear, loosening and osteolysis, and thereby optimize implant longevity. These alternatives include ceramic-onceramic bearings or ceramic head or highly polished cobalt-chrome femoral heads articulating with highly-cross linked polyethylene.

Another concern relates to primary diagnosis and the expecting of worse prognosis especially in patients with osteoarthritis after developmental dysplasia in comparison with those with idiopathic osteoarthritis. This may be associated at least partially with severity of dysplasia (Chougle et al. 2005). In fact, experienced surgeon and choice of implant could also play a role. Recent analysis from the Danish Hip Arthroplasty Registry failed to reveal substantial differences in revision risk between the above groups with follow-up of from 0.5 to 12 years postoperatively (Thillemann et al. 2008). Low revision rate has been observed in both cemented and cementless THA (Sochart and Porter 1997; Rozkydal et al. 2005). Rheumatoid arthritis does not require a special approach even in young patients. Regarding the choice of implant in these patients, a higher risk of revision was found for THAs with uncemented cups and cemented stems according in the Finnish Arthroplasty Registry (Eskelinen et al. 2006). However, the difference in survivorship between cemented and uncemented THAs disappeared in patients older than 55 years old (Makela et al. 2011).

\subsection{Operative procedures}

Surgically-related variables are those related to wear rate and those that influence the strength of implant-bone fixation. Wear rate can be influenced primarily by appropriate choice of implant and experienced surgical technique especially in terms of positioning of the cup and 
stem, assuming both the inappropriate bearing surfaces and/or incorrect positioning of the implants contribute to increased wear rate and risk of osteolysis development (Little et al. 2009). Surgical technique can also influence the strength of implant-bone interface. In this connection, any mistake during cementation can increase the risk of premature failure (Barrack, Mulroy et al. 1992). In support, it seems that high-volume surgeons can achieve lower revision rates than low-volume surgeons especially during the early period after the surgery (Losina et al. 2004).

\subsection{Life with total hip arthroplasty}

Charnley believed that THA is appropriate for older people with limited physical expectations (Charnley and Feagin 1973). Later on, younger and more active patients were indicated for prosthetic hip surgery and the expectations of these patients have increased continually. In fact, younger patients who are treated by THA wish to return to recreational and even competitive sports activity. At the same time, patient activity is considered one of the most important factors in the risk of failure especially via increased wear-rate of bearing surfaces (Schmalzried et al. 2000). By this way, those who experience high-levels of activity are at increased risk for failure due to aseptic loosening and osteolysis, and vice versa, lower impact activities are less likely to create conditions appropriate to loosening (Kilgus et al. 1991; Lubbeke et al. 2011). Regarding the stability of bone-implant interface, there may be a concern about cracks followed by fracture of the cement mantle in association with highimpact load. Surgeons should advise patients with THA that all high-impact activities could compromise the durability of their implant, especially in terms of accelerated wear and premature aseptic loosening. Another possible concern is risk of fall and direct contact during these sport activities that could lead to catastrophic failure, periprosthetic fracture or dislocation of THA. In addition, patients should know that return to a high-load level is not possible without achieving a very good muscle condition and also appropriate skills in their coordination beforehand as muscles can share the forces traversing an artificial joint and thus could prevent its damage. On the other hand, regular repetitive physical activities positively affect both the bone (in accordance with Wolff's law) and muscles near the artificial joint (Clifford and Mallon 2005; Wilson and Villar 2011).

\section{Conclusions (Gallo)}

Aseptic loosening is the leading cause of THA failure in the intermediate and long-term postoperative period. Causally it is associated with rapid wear rate inducing adverse host reaction to wear debris and to repetitive mechanical stresses and strains of the bone-implant interface. Here, we introduced the concept of failure of local tissue homeostasis as underlying the majority of processes leading to aseptic loosening. Given that the implant is stable in the second postoperative year and no gross pathology of bone-implant interface is seen on x-ray, then the fate of implant depends on the ability of host tissues (both, bone and soft tissues) to maintain homeostasis with minimum inflammation despite the occurrence of chronic mechanical and particle load. In this connection, the problem is the inability to maintain the balance at bone-implant interface and resolve inflammation in the periprosthetic tissues that can induce the excessive bone resorption around the implant (osteolysis) leading eventually to aseptic loosening. The increased knowledge of the mechanisms regulating the balance in periprosthetic tissues might open new avenues to prevent aseptic loosening and periprosthetic osteolysis. 


\section{List of abbreviations used in the chapter}

ALVAL Aseptic Lymphocyte-dominated Vasculitis-Associated Lesions

ARF

BMP

Activation-Reversal-Formation cycles (bone remodelation)

BMU

$\mathrm{CD}$

Bone Morphogenetic Protein

$\mathrm{CoC}$

Bone Multicellular Unit

DTH

Cluster of Differentiation

ECM

Ceramic on Ceramic

FBGCs

FGF

GM-CSF

HIF

Delayed-Type Hypersensitivity

HLA

ExtraCellular Matrix

HXLPE

Foreign Body Giant Cells

ICAM

IFN-ץ

IL-1 $\beta, 2$..

Fibroblast Growth Factor

IRAK

LPS

Granulocyte Macrophage Colony Stimulating Factor

MCP-1

Hypoxia-Induced Factor

M-CSF

MHC

MIP

Human Leukocyte Antigen (HLA DR+ is a MHC II class II cell surface receptor)

MoM

$\mathrm{MoP}$

Highly Cross-Linked PolyEthylene

$\mathrm{MPa}$

InterCellular Adhesion Molecule

Interferon gama

Interleukins

Interleukin-1-Receptor-Associated Kinase

LipoPolySaccharide

MSCs Mesenchymal Stromal/Stem Cells

NF-k $\beta$

Monocyte Chemoattractant Protein-1

NK cells

Macrophage Colony-Stimulating Factor

OPG

Major Histocompatibility Complex

PAMPS

Macrophage Inhibitory Protein

Metal on Metal

PMMA

Metal on Polyethylene

Megapascal (pressure unit)

PRRs

Nuclear Factor kappa beta

RANK

Natural Killer cells

Osteoprotegerin

RANKL

ROS

SLIM

SNP

SOCS

TGF

THA

TLRs

Pathogen-Associated Molecular Patterns

TNF-a Tumor Necrosis Factor alpha

TREG T-REGulatory lymphocytes

UHMWPE Ultra-High Molecular Weight PolyEthylene

VCAM Vascular Cell Adhesion Molecule

VEGF Vascular Endothelial Growth Factor 


\section{Acknowledgements}

This study was supported by Internal Grant Agency Ministry of Health, Czech Republic (NT/11049-5), the Sigrid Juselius Foundation, by the Helsinki University Central Hospital evo-funds, Finska Läkaresällskapet, Wilhelm och Else Stockmanns Stiftelse, ORTON Orthopaedic Hospital of the Invalid Foundation, National Doctoral Programme of Musculoskeletal Disorders and Biomaterials, Danish Council for Strategic Research, European Science Foundation “Regenerative Medicine” RNP, and by Grant 1R01AR05565004 from the National Institute of Health and the French Granting Agency E.F.M.C. Nº1.

We apologize to all authors whose work could not be cited due to space limitations.

\section{References}

Al-Saffar, N. and P. A. Revell (2000). "Differential expression of transforming growth factoralpha and macrophage colony-stimulating factor/colony-stimulating factor-1R (cfins) by multinucleated giant cells involved in pathological bone resorption at the site of orthopaedic implants." J Orthop Res 18(5): 800-7.

Amis, A. A. (1996). "Is polyethylene still the best prosthetic bearing surface?" J Bone Joint Surg Br 78(3): 345-8.

Anderson, J. M. (2009). In vitro and in vivo monocyte, macrophage, foreign body giant cell, and lymphocyte interactions with biomaterials. In: Biological interactions on material surfaces. Eds. D. A. Puleo, Bizios, R. Dordrecht, Springer: 225-244.

Anderson, J. M., A. Rodriguez, et al. (2008). "Foreign body reaction to biomaterials." Seminars in Immunology 20(2): 86-100.

Aspenberg, P., N. Basic, et al. (2000). "Reduced expression of BMP-3 due to mechanical loading: a link between mechanical stimuli and tissue differentiation." Acta Orthop Scand 71(6): 558-62.

Aspenberg, P., P. Wagner, et al. (2008). "Fixed or loose? Dichotomy in RSA data for cemented cups." Acta Orthop 79(4): 467-73.

Baggiolini, M. and I. Clark-Lewis (1992). "Interleukin-8, a chemotactic and inflammatory cytokine." FEBS Lett 307(1): 97-101.

Barrack, R. L., R. D. Mulroy, Jr., et al. (1992). "Improved cementing techniques and femoral component loosening in young patients with hip arthroplasty. A 12-year radiographic review." J Bone Joint Surg Br 74(3): 385-9.

Basko-Plluska, J. L., J. P. Thyssen, et al. (2011). "Cutaneous and systemic hypersensitivity reactions to metallic implants." Dermatitis 22(2): 65-79.

Beyersmann, D. and A. Hartwig (2008). "Carcinogenic metal compounds: recent insight into molecular and cellular mechanisms." Arch Toxicol 82(8): 493-512.

Bordignon, V., F. Palamara, et al. (2008). "Nickel, palladium and rhodium induced IFNgamma and IL-10 production as assessed by in vitro ELISpot-analysis in contact dermatitis patients." BMC Immunol 9: 19.

Boyle, W. J., W. S. Simonet, et al. (2003). "Osteoclast differentiation and activation." Nature 423(6937): 337-42.

Brasch, J., T. Henseler, et al. (1994). "Reproducibility of patch tests. A multicenter study of synchronous left-versus right-sided patch tests by the German Contact Dermatitis Research Group." J Am Acad Dermatol 31(4): 584-91. 
Buckley, C. D. (2011). "Why does chronic inflammation persist: An unexpected role for fibroblasts." Immunol Lett 138(1): 12-4.

Burke, D. W., D. O. O'Connor, et al. (1991). "Micromotion of cemented and uncemented femoral components." J Bone Joint Surg Br 73(1): 33-7.

Cadosch, D., M. S. Al-Mushaiqri, et al. (2010a). "Biocorrosion and uptake of titanium by human osteoclasts." J Biomed Mater Res A 95(4): 1004-10.

Cadosch, D., O. P. Gautschi, et al. (2010b). "Titanium induced production of chemokines CCL17/TARC and CCL22/MDC in human osteoclasts and osteoblasts." J Biomed Mater Res A 92(2): 475-83.

Cadosch, D., E. Chan, et al. (2009). "Metal is not inert: role of metal ions released by biocorrosion in aseptic loosening--current concepts." J Biomed Mater Res A 91(4): 1252-62.

Caicedo, M. S., R. Desai, et al. (2009). "Soluble and particulate Co-Cr-Mo alloy implant metals activate the inflammasome danger signaling pathway in human macrophages: a novel mechanism for implant debris reactivity." J Orthop Res 27(7): 847-54.

Clifford, P. E. and W. J. Mallon (2005). "Sports after total joint replacement." Clin Sports Med 24(1): 175-86.

Clohisy, J. C., E. Frazier, et al. (2003). "RANKL is an essential cytokine mediator of polymethylmethacrylate particle-induced osteoclastogenesis." J Orthop Res 21(2): 202-12.

Cook, D. N. (1996). "The role of MIP-1 alpha in inflammation and hematopoiesis." J Leukoc Biol 59(1): 61-6.

Corbett, K. L., E. Losina, et al. (2010). "Population-based rates of revision of primary total hip arthroplasty: a systematic review." PLoS One 5(10): e13520.

Del Buono, A., V. Denaro, et al. (2011). "Genetic susceptibility to aseptic loosening following total hip arthroplasty: a systematic review." Br Med Bull. Epub ahead of print Jun 7.

Dempsey, K. E., M. P. Riggio, et al. (2007). "Identification of bacteria on the surface of clinically infected and non-infected prosthetic hip joints removed during revision arthroplasties by $16 \mathrm{~S}$ rRNA gene sequencing and by microbiological culture." Arthritis Res Ther 9(3): R46.

Derbyshire, B., R. J. Prescott, et al. (2009). "Notes on the use and interpretation of radiostereometric analysis." Acta Orthop 80(1): 124-30.

Deshmane, S. L., S. Kremlev, et al. (2009). "Monocyte chemoattractant protein-1 (MCP-1): an overview." J Interferon Cytokine Res 29(6): 313-26.

Djaafar, S., D. D. Pierroz, et al. (2010). "Inhibition of T cell-dependent and RANKLdependent osteoclastogenic processes associated with high levels of bone mass in interleukin-15 receptor-deficient mice." Arthritis Rheum 62(11): 3300-10.

Dupuis, J. and C. J. O'Donnell (2007). "Interpreting results of large-scale genetic association studies: separating gold from fool's gold." JAMA 297(5): 529-31.

El-Warrak, A. O., M. Olmstead, et al. (2004). "An experimental animal model of aseptic loosening of hip prostheses in sheep to study early biochemical changes at the interface membrane." BMC Musculoskelet Disord 5: 7.

Engesaeter, L. B., S. A. Lie, et al. (2003). "Antibiotic prophylaxis in total hip arthroplasty: effects of antibiotic prophylaxis systemically and in bone cement on the revision 
rate of 22,170 primary hip replacements followed 0-14 years in the Norwegian Arthroplasty Register." Acta Orthop Scand 74(6): 644-51.

Engh, C. A., H. Ho, et al. (2011). "Osteolysis propensity among bilateral total hip arthroplasty patients." J Arthroplasty 26(4): 555-61.

Engh, C. A., J. P. Hooten, Jr., et al. (1995). "Evaluation of bone ingrowth in proximally and extensively porous-coated anatomic medullary locking prostheses retrieved at autopsy." J Bone Joint Surg Am 77(6): 903-10.

Eskelinen, A., P. Paavolainen, et al. (2006). "Total hip arthroplasty for rheumatoid arthritis in younger patients: 2,557 replacements in the Finnish Arthroplasty Register followed for 0-24 years." Acta Orthop 77(6): 853-65.

Fahlgren, A., M. P. Bostrom, et al. (2010). "Fluid pressure and flow as a cause of bone resorption." Acta Orthop 81(4): 508-16.

Faurschou, A., T. Menne, et al. (2011). "Metal allergen of the 21st century--a review on exposure, epidemiology and clinical manifestations of palladium allergy." Contact Dermatitis 64(4): 185-95.

Fritz, E. A., T. T. Glant, et al. (2002). "Titanium particles induce the immediate early stress responsive chemokines IL-8 and MCP-1 in osteoblasts." J Orthop Res 20(3): 490-8.

Fujii, J., Y. Yasunaga, et al. (2011). "Wear debris stimulates bone-resorbing factor expression in the fibroblasts and osteoblasts." Hip Int.

Fujishiro, T., D. J. Moojen, et al. (2011). "Perivascular and diffuse lymphocytic inflammation are not specific for failed metal-on-metal hip implants." Clin Orthop Relat Res 469(4): 1127-33.

Furmanski, J., M. Anderson, et al. (2009). "Clinical fracture of cross-linked UHMWPE acetabular liners." Biomaterials 30(29): 5572-82.

Gallo, J., V. Havranek, et al. (2010). "Male gender, Charnley class C, and severity of bone defects predict the risk for aseptic loosening in the cup of ABG I hip arthroplasty." BMC Musculoskelet Disord 11: 243.

Gallo, J., P. Kaminek, et al. (2002). "Particle disease. A comprehensive theory of periprosthetic osteolysis: a review." Biomed Pap Med Fac Univ Palacky Olomouc Czech Repub 146(2): 21-8.

Goldstein, I. M., D. Roos, et al. (1975). "Complement and immunoglobulins stimulate superoxide production by human leukocytes independently of phagocytosis." J Clin Invest 56(5): 1155-63.

Goodman, S. B., P. Huie, et al. (1998). "Cellular profile and cytokine production at prosthetic interfaces. Study of tissues retrieved from revised hip and knee replacements." J Bone Joint Surg Br 80(3): 531-9.

Goodman, S. B., R. C. Chin, et al. (1989). "A clinical-pathologic-biochemical study of the membrane surrounding loosened and nonloosened total hip arthroplasties." Clin Orthop Relat Res(244): 182-7.

Goodman, S. B. and T. Ma (2010). "Cellular chemotaxis induced by wear particles from joint replacements." Biomaterials 31(19): 5045-50.

Granchi, D., I. Amato, et al. (2005). "Molecular basis of osteoclastogenesis induced by osteoblasts exposed to wear particles." Biomaterials 26(15): 2371-9.

Greenfield, E. M., M. A. Beidelschies, et al. (2010). "Bacterial pathogen-associated molecular patterns stimulate biological activity of orthopaedic wear particles by activating cognate Toll-like receptors." J Biol Chem 285(42): 32378-84. 
Gross, T. S., K. A. King, et al. (2005). "Upregulation of osteopontin by osteocytes deprived of mechanical loading or oxygen." J Bone Miner Res 20(2): 250-6.

Hailer, N. P., R. A. Blaheta, et al. (2011). "Elevation of circulating HLA DR(+) CD8(+) T-cells and correlation with chromium and cobalt concentrations 6 years after metal-onmetal hip arthroplasty." Acta Orthop 82(1): 6-12.

Hailer, N. P., G. Garellick, et al. (2010). "Uncemented and cemented primary total hip arthroplasty in the Swedish Hip Arthroplasty Register." Acta Orthop 81(1): 34-41.

Hallab, N., K. Merritt, et al. (2001). "Metal sensitivity in patients with orthopaedic implants." J Bone Joint Surg Am 83-A(3): 428-36.

Hallab, N. J., S. Anderson, et al. (2004). "Immune responses correlate with serum-metal in metal-on-metal hip arthroplasty." J Arthroplasty 19(8 Suppl 3): 88-93.

Hallab, N. J., M. Caicedo, et al. (2008). "Th1 type lymphocyte reactivity to metals in patients with total hip arthroplasty." J Orthop Surg Res 3: 6.

Hallab, N. J. and J. J. Jacobs (2009). "Biologic effects of implant debris." Bull NYU Hosp Jt Dis 67(2): 182-8.

Hannouche, D., A. Zaoui, et al. (2011). "Thirty years of experience with alumina-on-alumina bearings in total hip arthroplasty." Int Orthop 35(2): 207-13.

Hilton, M. J., X. Tu, et al. (2008). "Notch signaling maintains bone marrow mesenchymal progenitors by suppressing osteoblast differentiation." Nat Med 14(3): 306-14.

Holt, G., C. Murnaghan, et al. (2007). "The Biology of Aseptic Osteolysis." Clin Orthop Relat Res 460: 240-252.

Hubbi, M. E., W. Luo, et al. (2011). "MCM Proteins Are Negative Regulators of HypoxiaInducible Factor 1." Mol Cell 42(5): 700-12.

Huss, R. S., J. I. Huddleston, et al. (2010). "Synovial tissue-infiltrating natural killer cells in osteoarthritis and periprosthetic inflammation." Arthritis Rheum 62(12): 3799-805.

Chanock, S. J., T. Manolio, et al. (2007). "Replicating genotype-phenotype associations." Nature 447(7145): 655-60.

Charnley, J. and J. A. Feagin (1973). "Low-friction arthroplasty in congenital subluxation of the hip." Clin Orthop Relat Res(91): 98-113.

Childs, L. M., J. J. Goater, et al. (2001). "Efficacy of etanercept for wear debris-induced osteolysis." J Bone Miner Res 16(2): 338-47.

Childs, L. M., E. P. Paschalis, et al. (2002). "In vivo RANK signaling blockade using the receptor activator of NF-kappaB:Fc effectively prevents and ameliorates wear debris-induced osteolysis via osteoclast depletion without inhibiting osteogenesis." J Bone Miner Res 17(2): 192-9.

Chougle, A., M. V. Hemmady, et al. (2005). "Severity of hip dysplasia and loosening of the socket in cemented total hip replacement. A long-term follow-up." J Bone Joint Surg Br 87(1): 16-20.

Illgen, R. L., 2nd, L. M. Bauer, et al. (2009). "Highly crosslinked vs conventional polyethylene particles: relative in vivo inflammatory response." J Arthroplasty 24(1): 117-24.

Ioannidis, J. P., T. A. Trikalinos, et al. (2006). "Implications of small effect sizes of individual genetic variants on the design and interpretation of genetic association studies of complex diseases." Am J Epidemiol 164(7): 609-14.

Jasty, M., W. J. Maloney, et al. (1990). "Histomorphological studies of the long-term skeletal responses to well fixed cemented femoral components." J Bone Joint Surg Am 72(8): 1220-9. 
Jin, Z. M., Fischer, J., Ingham E. (2006). Biotribology: material design, lubrication, and wear in artificial hip joints. In: Handbook of Lubrication and Tribology. Volume I Application and Maintenance. Ed. G. E. Totten. Boca Raton, FL, CRC Press.

Jones, L. C., M. Tucci, et al. (2006). "Macrophages and fibroblasts respond differently to PMMA particles and mechanical strain." Biomed Sci Instrum 42: 223-30.

Kilgus, D. J., F. J. Dorey, et al. (1991). "Patient activity, sports participation, and impact loading on the durability of cemented total hip replacements." Clin Orthop Relat $\operatorname{Res}(269): 25-31$.

Kim, K. J., S. Kotake, et al. (2001). "Osteoprotegerin inhibits in vitro mouse osteoclast formation induced by joint fluid from failed total hip arthroplasty." J Biomed Mater Res 58(4): 393-400.

Knowles, H. J. and N. A. Athanasou (2009). "Acute hypoxia and osteoclast activity: a balance between enhanced resorption and increased apoptosis." J Pathol 218(2): 256-64.

Konttinen, Y. T., M. Takagi, et al. (2001). "Acid attack and cathepsin K in bone resorption around total hip replacement prosthesis." J Bone Miner Res 16(10): 1780-6.

Konttinen, Y. T., D. Zhao, et al. (2005). "The microenvironment around total hip replacement prostheses." Clin Orthop Relat Res(430): 28-38.

Koreny, T., M. Tunyogi-Csapo, et al. (2006). "The role of fibroblasts and fibroblast-derived factors in periprosthetic osteolysis." Arthritis Rheum 54(10): 3221-32.

Korhonen, R. K., A. Koistinen, et al. (2005). "The effect of geometry and abduction angle on the stresses in cemented UHMWPE acetabular cups--finite element simulations and experimental tests." Biomed Eng Online 4(1): 32.

Koulouvaris, P., K. Ly, et al. (2008). "Expression profiling reveals alternative macrophage activation and impaired osteogenesis in periprosthetic osteolysis." J Orthop Res 26(1): 106-16.

Kumar, H., T. Kawai, et al. (2011). "Pathogen recognition by the innate immune system." Int Rev Immunol 30(1): 16-34.

Kumazawa, R., F. Watari, et al. (2002). "Effects of Ti ions and particles on neutrophil function and morphology." Biomaterials 23(17): 3757-64.

Kurtz, S., K. Ong, et al. (2007). "Projections of primary and revision hip and knee arthroplasty in the United States from 2005 to 2030." J Bone Joint Surg Am 89(4): 780-5.

Kurtz, S. M., H. A. Gawel, et al. (2011). "History and Systematic Review of Wear and Osteolysis Outcomes for First-generation Highly Crosslinked Polyethylene." Clin Orthop Relat Res (469): 2262-77.

Lahdeoja, T., J. Pajarinen, et al. (2010). "Toll-like receptors and aseptic loosening of hip endoprosthesis-a potential to respond against danger signals?" J Orthop Res 28(2): 184-90.

Landgraeber, S., S. Jaeckel, et al. (2009). "Pan-caspase inhibition suppresses polyethylene particle-induced osteolysis." Apoptosis 14(2): 173-81.

Lappalainen, R., M. Selenius, et al. (2003). "Reduction of wear in total hip replacement prostheses by amorphous diamond coatings." J Biomed Mater Res B Appl Biomater 66(1): 410-3.

Lazarov, A., M. David, et al. (2007). "Comparison of reactivity to allergens using the TRUE Test and IQ chamber system." Contact Dermatitis 56(3): 140-5.

Little, N. J., C. A. Busch, et al. (2009). "Acetabular polyethylene wear and acetabular inclination and femoral offset." Clin Orthop Relat Res 467(11): 2895-900. 
Losina, E., J. Barrett, et al. (2004). "Early failures of total hip replacement: effect of surgeon volume." Arthritis Rheum 50(4): 1338-43.

Lubbeke, A., G. Garavaglia, et al. (2011). "Influence of patient activity on femoral osteolysis at five and ten years following hybrid total hip replacement." J Bone Joint Surg Br 93(4): 456-63.

Ma, J., T. Chen, et al. (2003). "Regulation of macrophage activation." Cell Mol Life Sci 60(11): 2334-46.

Ma, T., S. G. Ortiz, et al. (2009). "In vivo murine model of continuous intramedullary infusion of particles--a preliminary study." J Biomed Mater Res B Appl Biomater 88(1): 250-3.

Maitra, R., C. C. Clement, et al. (2009). "Endosomal damage and TLR2 mediated inflammasome activation by alkane particles in the generation of aseptic osteolysis." Mol Immunol 47(2-3): 175-84.

Makela, K. T., A. Eskelinen, et al. (2011). "Cemented versus cementless total hip replacements in patients fifty-five years of age or older with rheumatoid arthritis." J Bone Joint Surg Am 93(2): 178-86.

Mandelin, J., T. F. Li, et al. (2005a). "Interface tissue fibroblasts from loose total hip replacement prosthesis produce receptor activator of nuclear factor-kappaB ligand, osteoprotegerin, and cathepsin K." J Rheumatol 32(4): 713-20.

Mandelin, J., T. F. Li, et al. (2003). "Imbalance of RANKL/RANK/OPG system in interface tissue in loosening of total hip replacement." J Bone Joint Surg Br 85(8): 1196-201.

Mandelin, J., M. Liljestrom, et al. (2005b). "Pseudosynovial fluid from loosened total hip prosthesis induces osteoclast formation." J Biomed Mater Res B Appl Biomater 74(1): 582-8.

Matzinger, P. (2007). "Friendly and dangerous signals: is the tissue in control?" Nat Immunol 8(1): 11-3.

Matzinger, P. and T. Kamala (2011). "Tissue-based class control: the other side of tolerance." Nat Rev Immunol 11(3): 221-30.

McEvoy, A., M. Jeyam, et al. (2002). "Synergistic effect of particles and cyclic pressure on cytokine production in human monocyte/macrophages: proposed role in periprosthetic osteolysis." Bone 30(1): 171-7.

McGregor, D. B., R. A. Baan, et al. (2000). "Evaluation of the carcinogenic risks to humans associated with surgical implants and other foreign bodies - a report of an IARC Monographs Programme Meeting. International Agency for Research on Cancer." Eur J Cancer 36(3): 307-13.

Medzhitov, R. (2010). "Inflammation 2010: new adventures of an old flame." Cell 140(6): 7716.

Medzhitov, R. and T. Horng (2009). "Transcriptional control of the inflammatory response." Nat Rev Immunol 9(10): 692-703.

Menten, P., A. Wuyts, et al. (2002). "Macrophage inflammatory protein-1." Cytokine Growth Factor Rev 13(6): 455-81.

Mjoberg, B. (1994). "Theories of wear and loosening in hip prostheses. Wear-induced loosening vs loosening-induced wear--a review." Acta Orthop Scand 65(3): 361-71.

Nakashima, Y., D. H. Sun, et al. (1999). "Induction of macrophage C-C chemokine expression by titanium alloy and bone cement particles." J Bone Joint Surg Br 81(1): 155-62.

Nalepka, J. L., M. J. Lee, et al. (2006). "Lipopolysaccharide found in aseptic loosening of patients with inflammatory arthritis." Clin Orthop Relat Res 451: 229-35. 
Nathan, C. and A. Ding (2010). "Nonresolving inflammation." Cell 140(6): 871-82.

$\mathrm{Ng}$, V. Y., A. V. Lombardi, Jr., et al. (2011). "Perivascular lymphocytic infiltration is not limited to metal-on-metal bearings." Clin Orthop Relat Res 469(2): 523-9.

Ogino, D., H. Kawaji, et al. (2008). "Total hip replacement in patients eighty years of age and older." J Bone Joint Surg Am 90(9): 1884-90.

Pajarinen, J., E. Cenni, et al. (2010). "Profile of toll-like receptor-positive cells in septic and aseptic loosening of total hip arthroplasty implants." J Biomed Mater Res A 94(1): 84-92.

Pearl, J. I., T. Ma, et al. (2011). "Role of the Toll-like receptor pathway in the recognition of orthopedic implant wear-debris particles." Biomaterials 32(24): 5535-42.

Pedersen, A. B., S. P. Johnsen, et al. (2005). "Total hip arthroplasty in Denmark: incidence of primary operations and revisions during 1996-2002 and estimated future demands." Acta Orthop 76(2): 182-9.

Perez-Losada, J. and A. Balmain (2003). "Stem-cell hierarchy in skin cancer." Nat Rev Cancer 3(6): 434-43.

Proudfoot, A. E. (2002). "Chemokine receptors: multifaceted therapeutic targets." Nat Rev Immunol 2(2): 106-15.

Purdue, P. E., P. Koulouvaris, et al. (2007). "The cellular and molecular biology of periprosthetic osteolysis." Clin Orthop Relat Res 454: 251-61.

Rasanen, L. and M. L. Tuomi (1992). "Diagnostic value of the lymphocyte proliferation test in nickel contact allergy and provocation in occupational coin dermatitis." Contact Dermatitis 27(4): 250-4.

Reilkoff, R. A., R. Bucala, et al. (2011). "Fibrocytes: emerging effector cells in chronic inflammation." Nat Rev Immunol 11(6): 427-35.

Ren, P. G., Z. Huang, et al. (2010). "Surveillance of systemic trafficking of macrophages induced by UHMWPE particles in nude mice by noninvasive imaging." J Biomed Mater Res A 94(3): 706-11.

Ren, P. G., A. Irani, et al. (2011). "Continuous infusion of UHMWPE particles induces increased bone macrophages and osteolysis." Clin Orthop Relat Res 469(1): 113-22.

Roder, C., B. Bach, et al. (2010). "Obesity, age, sex, diagnosis, and fixation mode differently affect early cup failure in total hip arthroplasty: a matched case-control study of 4420 patients." J Bone Joint Surg Am 92(10): 1954-63.

Rozkydal, Z., P. Janicek, et al. (2005). "Total hip replacement with the CLS expansion shell and a structural femoral head autograft for patients with congenital hip disease." J Bone Joint Surg Am 87(4): 801-7.

Santavirta, S., Takagi, M., Gomez-Barrena, E., Nevalainen, J., Lassus, M., Salo, J., Konttinen, Y.T. (1999). "Studies of host response to orthopedic implants and biomaterials." J Long Term Eff Med Implants 9: 67-76.

Schmalzried, T. P., E. F. Shepherd, et al. (2000). "The John Charnley Award. Wear is a function of use, not time." Clin Orthop Relat Res(381): 36-46.

Schmalzried, T. P., E. S. Szuszczewicz, et al. (1998). "Quantitative assessment of walking activity after total hip or knee replacement." J Bone Joint Surg Am 80(1): 54-9.

Sierra, J. M., S. Garcia, et al. (2011). "Relationship between the degree of osteolysis and cultures obtained by sonication of the prostheses in patients with aseptic loosening of a hip or knee arthroplasty." Arch Orthop Trauma Surg. Epub ahead of print May 11. 
Sochart, D. H. and M. L. Porter (1997). "The long-term results of Charnley low-friction arthroplasty in young patients who have congenital dislocation, degenerative osteoarthrosis, or rheumatoid arthritis." J Bone Joint Surg Am 79(11): 1599-617.

St Pierre, C. A., M. Chan, et al. (2010). "Periprosthetic osteolysis: characterizing the innate immune response to titanium wear-particles." J Orthop Res 28(11): 1418-24.

Stanley, K. T., C. VanDort, et al. (2006). "Immunocompetent properties of human osteoblasts: interactions with T lymphocytes." J Bone Miner Res 21(1): 29-36.

Summer, B., C. Paul, et al. (2010). "Nickel (Ni) allergic patients with complications to Ni containing joint replacement show preferential IL-17 type reactivity to Ni." Contact Dermatitis 63(1): 15-22.

Sun, D. H., M. C. Trindade, et al. (2003). "Human serum opsonization of orthopedic biomaterial particles: protein-binding and monocyte/macrophage activation in vitro." J Biomed Mater Res A 65(2): 290-8.

Takagi, M., S. Santavirta, et al. (2001). "High-turnover periprosthetic bone remodeling and immature bone formation around loose cemented total hip joints." J Bone Miner Res 16(1): 79-88.

Takagi, M., Y. Tamaki, et al. (2007). "Toll-like receptors in the interface membrane around loosening total hip replacement implants." J Biomed Mater Res A 81(4): 1017-26.

Takahashi, N., B. R. MacDonald, et al. (1986). "Recombinant human transforming growth factor-alpha stimulates the formation of osteoclast-like cells in long-term human marrow cultures." J Clin Invest 78(4): 894-8.

Taki, N., J. M. Tatro, et al. (2007). "Comparison of the roles of IL-1, IL-6, and TNFalpha in cell culture and murine models of aseptic loosening." Bone 40(5): 1276-83.

Tamaki, Y., Y. Takakubo, et al. (2009). "Increased expression of toll-like receptors in aseptic loose periprosthetic tissues and septic synovial membranes around total hip implants." J Rheumatol 36(3): 598-608.

Tarala, M., D. Janssen, et al. (2011). "Balancing incompatible endoprosthetic design goals: a combined ingrowth and bone remodeling simulation." Med Eng Phys 33(3): 374-80.

Theill, L. E., W. J. Boyle, et al. (2002). "RANK-L and RANK: T cells, bone loss, and mammalian evolution." Annu Rev Immunol 20: 795-823.

Thillemann, T. M., A. B. Pedersen, et al. (2008). "Implant survival after primary total hip arthroplasty due to childhood hip disorders: results from the Danish Hip Arthroplasty Registry." Acta Orthop 79(6): 769-76.

Thillemann, T. M., A. B. Pedersen, et al. (2010a). "Postoperative use of bisphosphonates and risk of revision after primary total hip arthroplasty: a nationwide population-based study." Bone 46(4): 946-51.

Thillemann, T. M., A. B. Pedersen, et al. (2010b). "The risk of revision after primary total hip arthroplasty among statin users: a nationwide population-based nested casecontrol study." J Bone Joint Surg Am 92(5): 1063-72.

Thyssen, J. P., A. Linneberg, et al. (2007). "The epidemiology of contact allergy in the general population--prevalence and main findings." Contact Dermatitis 57(5): 287-99.

Thyssen, J. P. and T. Menne (2010). "Metal allergy--a review on exposures, penetration, genetics, prevalence, and clinical implications." Chem Res Toxicol 23(2): 309-18.

Thyssen, J. P., T. Menne, et al. (2011a). "Pragmatic approach to the clinical work-up of patients with putative allergic disease to metallic orthopaedic implants before and after surgery." Br J Dermatol 164(3): 473-8. 
Thyssen, J. P., W. Uter, et al. (2011b). "The EU Nickel Directive revisited--future steps towards better protection against nickel allergy." Contact Dermatitis 64(3): 121-5.

Tuan, R. S., F. Y. Lee, et al. (2008). "What are the local and systemic biologic reactions and mediators to wear debris, and what host factors determine or modulate the biologic response to wear particles?" J Am Acad Orthop Surg 16 Suppl 1: S42-8.

Ulrich-Vinther, M., E. E. Carmody, et al. (2002). "Recombinant adeno-associated virusmediated osteoprotegerin gene therapy inhibits wear debris-induced osteolysis." J Bone Joint Surg Am 84-A(8): 1405-12.

Ulrich, S. D., T. M. Seyler, et al. (2008). "Total hip arthroplasties: What are the reasons for revision?" Int Orthop 32(5): 597-604.

Uter, W. J., J. Geier, et al. (1996). "Good clinical practice in patch testing: readings beyond day 2 are necessary: a confirmatory analysis. Members of the Information Network of Departments of Dermatology." Am J Contact Dermat 7(4): 231-7.

Valledor, A. F., M. Comalada, et al. (2010). "Macrophage proinflammatory activation and deactivation: a question of balance." Adv Immunol 108: 1-20.

Vermes, C., R. Chandrasekaran, et al. (2001). "The effects of particulate wear debris, cytokines, and growth factors on the functions of MG-63 osteoblasts." J Bone Joint Surg Am 83-A(2): 201-11.

Vocanson, M., A. Hennino, et al. (2009). "Effector and regulatory mechanisms in allergic contact dermatitis." Allergy 64(12): 1699-714.

Wahlberg, J. E., Lindberg, M. (2006). Patch test. Contact Dermatitis. P. J. Frosch, Menne, T., Lepoittevin, J-P. Berlin, Springer.

Watters, T. S., D. M. Cardona, et al. (2010). "Aseptic lymphocyte-dominated vasculitisassociated lesion: a clinicopathologic review of an underrecognized cause of prosthetic failure." Am J Clin Pathol 134(6): 886-93.

Weyand, C. M., A. Geisler, et al. (1998). "Oligoclonal T-cell proliferation and interferongamma production in periprosthetic inflammation." Lab Invest 78(6): 677-85.

Whyte, C. S., E. T. Bishop, et al. (2011). "Suppressor of cytokine signaling (SOCS)1 is a key determinant of differential macrophage activation and function." J Leukoc Biol. Epub ahead of print May 31.

Wilkinson, D. S., S. Fregert, et al. (1970). "Terminology of contact dermatitis." Acta Derm Venereol 50(4): 287-92.

Wilkinson, J. M., A. G. Wilson, et al. (2003). "Variation in the TNF gene promoter and risk of osteolysis after total hip arthroplasty." J Bone Miner Res 18(11): 1995-2001.

Willert, H. G., J. Ludwig, et al. (1974). "Reaction of bone to methacrylate after hip arthroplasty: a long-term gross, light microscopic, and scanning electron microscopic study." J Bone Joint Surg Am 56(7): 1368-82.

Willert, H. G. and M. Semlitsch (1977). "Reactions of the articular capsule to wear products of artificial joint prostheses." J Biomed Mater Res 11(2): 157-64.

Wilson, M. J. and R. N. Villar (2011). "Hip replacement in the athlete: is there a role?" Knee Surg Sports Traumatol Arthrosc 19(9): 1524-30.

Zolotarevova, E., G. Entlicher, et al. (2010). "Distribution of polyethylene wear particles and bone fragments in periprosthetic tissue around total hip joint replacements." Acta Biomater 6(9): 3595-600. 


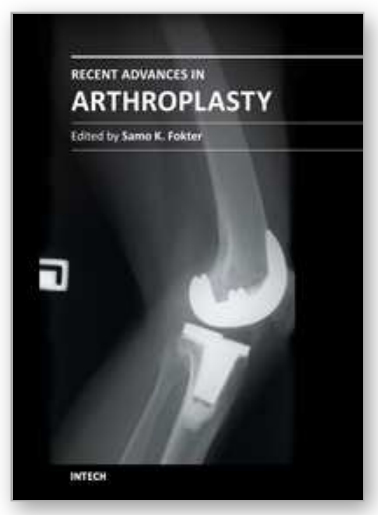

\author{
Recent Advances in Arthroplasty \\ Edited by Dr. Samo Fokter
}

ISBN 978-953-307-990-5

Hard cover, 614 pages

Publisher InTech

Published online 27, January, 2012

Published in print edition January, 2012

The purpose of this book was to offer an overview of recent insights into the current state of arthroplasty. The tremendous long term success of Sir Charnley's total hip arthroplasty has encouraged many researchers to treat pain, improve function and create solutions for higher quality of life. Indeed and as described in a special chapter of this book, arthroplasty is an emerging field in the joints of upper extremity and spine. However, there are inborn complications in any foreign design brought to the human body. First, in the chapter on infections we endeavor to provide a comprehensive, up-to-date analysis and description of the management of this difficult problem. Second, the immune system is faced with a strange material coming in huge amounts of micro-particles from the tribology code. Therefore, great attention to the problem of aseptic loosening has been addressed in special chapters on loosening and on materials currently available for arthroplasty.

\title{
How to reference
}

In order to correctly reference this scholarly work, feel free to copy and paste the following:

Jiri Gallo, Yrjö T. Konttinen, Stuart B. Goodman, Jacob P. Thyssen, Emmanuel Gibon, Jukka Pajarinen, Yuya Takakubo, Peter Schalock, Zygmunt Mackiewicz, Eemeli Jämsen, Martin Petrek, Rihard Trebse, Andrei Coer and Michiaki Takagi (2012). Aseptic Loosening of Total Hip Arthroplasty as a Result of Local Failure of Tissue Homeostasis, Recent Advances in Arthroplasty, Dr. Samo Fokter (Ed.), ISBN: 978-953-307-990-5, InTech, Available from: http://www.intechopen.com/books/recent-advances-in-arthroplasty/aseptic-loosening-of-totalhip-arthroplasty-as-a-result-of-local-failure-of-tissue-homeostasis

\section{INTECH}

open science | open minds

\section{InTech Europe}

University Campus STeP Ri

Slavka Krautzeka 83/A

51000 Rijeka, Croatia

Phone: +385 (51) 770447

Fax: +385 (51) 686166

www.intechopen.com

\section{InTech China}

Unit 405, Office Block, Hotel Equatorial Shanghai

No.65, Yan An Road (West), Shanghai, 200040, China

中国上海市延安西路65号上海国际贵都大饭店办公楼 405 单元

Phone: +86-21-62489820

Fax: $+86-21-62489821$ 
(C) 2012 The Author(s). Licensee IntechOpen. This is an open access article distributed under the terms of the Creative Commons Attribution 3.0 License, which permits unrestricted use, distribution, and reproduction in any medium, provided the original work is properly cited. 